\title{
SECOND-ORDER SUFFICIENT OPTIMALITY CONDITIONS FOR CONTROL PROBLEMS WITH LINEARLY INDEPENDENT GRADIENTS OF CONTROL CONSTRAINTS
}

\begin{abstract}
Nikolai P. OsmolovskiI ${ }^{1,2,3}$
Abstract. Second-order sufficient conditions of a bounded strong minimum are derived for optimal control problems of ordinary differential equations with initial-final state constraints of equality and inequality type and control constraints of inequality type. The conditions are stated in terms of quadratic forms associated with certain tuples of Lagrange multipliers. Under the assumption of linear independence of gradients of active control constraints they guarantee the bounded strong quadratic growth of the so-called "violation function". Together with corresponding necessary conditions they constitute a no-gap pair of conditions.
\end{abstract}

Mathematics Subject Classification. 49K15.

Received November 28, 2009. Revised November 27, 2010.

Published online June 22, 2011.

\section{INTRODUCTION}

In this paper we discuss sufficient second order conditions for bounded strong minimum in optimal control problems of ordinary differential equations with control constraints and constraints on the initial-final state. There exists an extensive literature on this subject, cf. Bonnans and Hermant [1], Bonnans and Shapiro [3], Bonnans and Osmolovskii [2], Levitin et al. [8], Malanowski [9,10], Maurer [11], Maurer and Pickenhain [12], Milyutin and Osmolovskii [17], Osmolovskii [19-22], Zeidan [23,24] and further literature cited in these papers. The no-gap second order conditions of Pontryagin and bounded strong minima in optimal control problems with initial-final state constraints and mixed state-control constraints, satisfying the hypothesis of linear independence of gradients (LIG) w.r.t. control of active mixed constraints, were formulated by the author in [8], pp. 155-156, but the proofs (written later in $[19,20]$ ) were not published. The aim of this paper is to publish the proofs of sufficient conditions [8] (partially modified in 2007-2008). For simplicity, we consider a less general problem than in [8], replacing mixed constraints by control constraints.

Recently, the same problem, as in the present paper, was analyzed by Bonnans and Osmolovskii in [2] under the hypothesis of positive linear independence (i.e., linear independence with nonnegative coefficients) of gradients (PLIG) of active control constraints. The no-gap second order conditions of Pontryagin and bounded

Keywords and phrases. Pontryagin's principle, critical cone, quadratic form, second order sufficient condition, quadratic growth, Hoffman's error bound.

1 Systems Research Institute, ul. Newelska 6, 01-447 Warszawa, Poland.

2 Politechnika Radomska, ul. Malczewskiego 20A, 26-600 Radom, Poland.

3 University of Natural Sciences and Humanities in Siedlce, ul. 3 Maja 54, 08-110 Siedlce, Poland. osmolovski@uph.edu.pl 
strong minima, similar to conditions [8], were obtained for this problem. Although the assumption of PLIG is weaker than the assumption of LIG, the results, obtained in [2], cannot be considered as a generalization of results [8], since in [2] we additionally assumed the Mangasarian-Fromovitz type condition for endpoint constraints and the so-called "restoration property" for these constraints. Hopefully, it would be possible to prove the same results, as in [2], under the PLIG hypothesis, but without two mentioned additional assumptions. The present publication could be an important step in this direction.

The paper is organized as follows. Section 2 sets the problem, recalls the concepts of bounded strong, Pontryagin and weak minima and gives the formulations of the first order necessary conditions of Pontryagin and weak minima, namely Pontryagin's principle and local Pontryagin's principle. In Section 3, for the cost functional, we define the "bounded strong growth condition of the order $\gamma$ ", where $\gamma$ is a continuous functional (in the space of variations $\delta w=(\delta u, \delta y)$ ) equal to $\|\delta y\|_{\infty}^{2}+\int_{0}^{T}|\delta u|^{2} \mathrm{~d} t$ in some neighborhood of zero and positive outside of this neighborhood. This condition implies a bounded strong minimum. We also define the violation function of the optimal control problem and the notion of the bounded strong $\gamma$-sufficiency (as the bounded strong growth condition of the order $\gamma$ for the violation function). The latter implies the bounded strong growth of the order $\gamma$ for the cost functional (and hence a bounded strong minimum). In Section 4, for the reference point $w=(x, u)$, we introduce the quadratic form $\Omega$ (the second variation of the Lagrange function) and the critical cone $\mathcal{K}$. We formulate a sufficient condition for a bounded strong $\gamma$-sufficiency in terms of $\Omega$ and $\mathcal{K}$. It should be noted that this condition implies the quadratic growth of the Hamiltonian, from which the continuity of the optimal control follows [2]. Sections 5-11 are devoted to the proofs. In Section 5 we define the set of Pontryagin's sequences $\Pi$ related to the notion of Pontryagin minimum and the so-called basic constant $C_{\gamma}$ for $\Pi$. We prove that the inequality $C_{\gamma}>0$ implies the bounded strong $\gamma$-sufficiency. Hence the positivity of any lower bound for $C_{\gamma}$ also implies the bounded strong $\gamma$-sufficiency. In the next sections, we obtain several successive lower bounds for $C_{\gamma}$, pursuing the goal to find the lower bound defined in the most simple way. In Section 7 we introduce a concept of support of the critical cone and show that the first order approximations of the endpoint constraints possesses Hoffman's error bound on the support. This fact plays a crucial role in the proof of sufficient optimality conditions and allows us to obtain in Section 9 (which completes the proof) a lower bound for $C_{\gamma}$ formulated in terms of the quadratic form $\Omega$ and the critical cone $\mathcal{K}$. The proofs of several basic lemmas are gathered in Section 10. In Section 11 we prove abstract lemmas (used earlier in Sect. 10) concerning compatibility of a system of linear inequalities on a convex cone and Hoffman's type [7] upper bounds for the distance from the origin to the set of solutions of such system on the cone. We also discuss an abstract notion of support of critical cone.

The paper is self-contained and formally does not use results of other papers. But it should be noted that the main idea of the proof (of sufficient optimality conditions), related to the role of the basic constant $C_{\gamma}$, first appeared in the abstract theory of higher order conditions published in $[8,14-16]$. We use notation introduced in these papers.

\section{Pontryagin And Bounded strong minima. First order necessary Conditions}

Consider the following optimal control problem on a fixed interval $[0, T]$ :

$$
\begin{aligned}
& \dot{y}(t)=f(u(t), y(t)) \text { for a.a. } t \in[0, T], \\
& u(t) \in U, \quad \text { for a.a. } t \in[0, T], \\
& \phi_{i}(y(0), y(T)) \leq 0, i=1, \ldots, r_{1}, \\
& \phi_{i}(y(0), y(T))=0, i=r_{1}+1, \ldots, r \\
& J(u, y):=\phi_{0}(y(0), y(T)) \rightarrow \min ,
\end{aligned}
$$

where $f: \mathbb{R}^{m} \times \mathbb{R}^{n} \rightarrow \mathbb{R}^{n}$ and $\phi_{i}: \mathbb{R}^{n} \times \mathbb{R}^{n} \rightarrow \mathbb{R}, i=0, \ldots, r$ are twice continuously differentiable $\left(C^{2}\right)$ mappings, $U$ is a closed subset of $\mathbb{R}^{m}$. Denote by $\mathcal{U}:=L^{\infty}\left(0, T ; \mathbb{R}^{m}\right)$ and $\mathcal{Y}:=W^{1,1}\left(0, T ; \mathbb{R}^{n}\right)$ the control and state space (where $W^{1,1}\left(0, T ; \mathbb{R}^{n}\right)$ is the space of absolutely continuous functions from $[0, T]$ to $\left.\mathbb{R}^{n}\right)$. We 
consider problem (2.1)-(2.5) in the space $\mathcal{W}:=\mathcal{U} \times \mathcal{Y}$, and we refer to this problem as problem $(P)$. Define the norm of element $w=(u, y) \in \mathcal{W}$ by $\|w\|_{\mathcal{W}}:=\|u\|_{\infty}+\|y\|_{1,1}=\operatorname{ess}_{\sup } \sup _{[0, T]}|u(t)|+|y(0)|+\int_{0}^{T}|\dot{y}(t)| \mathrm{d} t$. Elements of $\mathcal{W}$ satisfying (2.1)-(2.4) are said to be feasible. The set of feasible points is denoted by $F(P)$. We shall use abbreviations $y_{0}:=y(0), y_{T}:=y(T), \eta:=\left(y_{0}, y_{T}\right)$.

It is well known that any control problem with a cost functional in the integral form $J=\int_{0}^{T} \mathcal{F}(u, y) \mathrm{d} t$ can be represented in the endpoint form by introducing a new state variable $z$ defined by the state equation $\dot{z}=\mathcal{F}(u, y)$, $z(0)=0$. This yields the cost functional $J=z(T)$. The new variable $z$ is called unessential component in the augmented problem. The general definition of an unessential component [17], p. 290, is as follows. The state variable $y_{i}$, i.e., the $i$ th component of the state vector $y$ is called unessential if the function $f$ does not depend on $y_{i}$ and the functions $\phi_{j}, j=0,1, \ldots, r$ are affine in $y_{i 0}:=y_{i}(0)$ and $y_{i T}:=y_{i}(T)$. Let $\underline{y}$ denote the vector of all essential components of the state vector $y$. We introduce two concepts of minimum.

Definition 2.1. Following [8], p. 156, and [17], p. 291, we say that $w^{0}=\left(u^{0}, y^{0}\right) \in F(P)$ is a bounded strong minimum if $J\left(w^{0}\right) \leq J\left(w_{k}\right)$ for large enough $k$ for any sequence $w_{k} \in F(P)$, bounded in $\mathcal{W}$, such that $\underline{y}_{k} \rightarrow \underline{y}^{0}$ uniformly and $y_{k}(0) \rightarrow y^{0}(0)$.

Definition 2.2. We say that $w^{0} \in F(P)$ is a Pontryagin minimum (see [8], p. 156, and [17], pp. 2-3) if $J\left(w^{0}\right) \leq J\left(w_{k}\right)$ for large enough $k$ for any sequence $w_{k} \in F(P)$, bounded in $\mathcal{W}$, such that $y_{k} \rightarrow y^{0}$ uniformly and $\left\|u_{k}-u^{0}\right\|_{1} \rightarrow 0$, where $\|u\|_{1}=\int_{0}^{T}|u(t)| \mathrm{d} t$.

Equivalently, $w^{0}$ is a bounded strong minimum iff for any $M>0$, there exists $\varepsilon>0$ such that if $w \in F(P)$ is such that $\|u\|_{\infty} \leq M,\left\|\underline{y}-\underline{y}^{0}\right\|_{\infty} \leq \varepsilon$, and $\left|y(0)-y^{0}(0)\right|<\varepsilon$, we have $J\left(w^{0}\right) \leq J(w)$. A point $w^{0}$ is a Pontryagin minimum iff for any $M>0$, there exists $\varepsilon>0$ such that if $w \in F(P)$ is such that $\|u\|_{\infty} \leq M$, $\left\|y-y^{0}\right\|_{\infty} \leq \varepsilon$, and $\left\|u-u^{0}\right\|_{1}<\varepsilon$, we have $J\left(w^{0}\right) \leq J(w)$. Obviously, a bounded strong minimum implies a Pontryagin minimum.

The strict bounded strong (Pontryagin) minimum is defined in a similar way. The only two distinctions are that the nonstrict inequality $J\left(w^{0}\right) \leq J\left(w_{k}\right)$ (in Defs. 2.1 and 2.2) is replaced by the corresponding strict inequality and the sequence $w_{k} \in F(P)$ is assumed to contain no terms equal to $w^{0}$.

Let us recall the formulation of Pontryagin's principle at a point $w \in F(P)$. Denote by $\mathbb{R}^{n *}$ the dual to $\mathbb{R}^{n}$ identified with the set of $n$-dimensional row vectors. Set

$$
\varphi^{\mu}\left(y_{0}, y_{T}\right)=\varphi\left(y_{0}, y_{T}, \mu\right):=\sum_{i=0}^{r} \mu_{i} \phi_{i}\left(y_{0}, y_{T}\right)
$$

where $y_{0}=y(0), y_{T}=y(T), \mu=\left(\mu_{0}, \ldots, \mu_{r}\right) \in \mathbb{R}^{(r+1) *}$. Consider the Hamiltonian function $H: \mathbb{R}^{m} \times \mathbb{R}^{n} \times$ $\mathbb{R}^{n *} \rightarrow \mathbb{R}$ defined by

$$
H(u, y, p)=p f(u, y) .
$$

Let $W^{1, \infty}\left(0, T, \mathbb{R}^{n *}\right)$ denote the space of Lipschitz continuous functions from $[0, T]$ to $\mathbb{R}^{n *}$. By the costate associated with $\mu \in \mathbb{R}^{(r+1) *}$ we understand the solution $p=p^{\mu} \in W^{1, \infty}\left(0, T, \mathbb{R}^{n *}\right)$ (whenever it exists) of

$$
\begin{aligned}
-\dot{p}(t) & =H_{y}(u(t), y(t), p(t)), \quad \text { a.a. } t \in[0, T] ; \\
p(0) & =-\varphi_{y_{0}}^{\mu}(y(0), y(T)) ; \quad p(T)=\varphi_{y_{T}}^{\mu}(y(0), y(T)) .
\end{aligned}
$$

Definition 2.3. We say that $w=(u, y) \in F(P)$ satisfies Pontryagin's principle if there exist a nonzero $\mu \in \mathbb{R}^{(r+1) *}$ and $p \in W^{1, \infty}\left(0, T, \mathbb{R}^{n *}\right)$ such that $(2.8)$ holds and

$$
\begin{aligned}
& \mu_{i} \geq 0, \quad i=0, \ldots, r_{1}, \quad \mu_{i} \phi_{i}(y(0), y(T))=0, \quad i=1, \ldots, r_{1}, \\
& H(u(t), y(t), p(t)) \leq H(v, y(t), p(t)), \quad \text { for all } v \in U, \text { a.a. } t \in(0, T) .
\end{aligned}
$$


The following theorem holds (see, e.g., [13], pp. 17-19, and [17], pp. 24-25, 150).

Theorem 2.4. A Pontryagin minimum satisfies Pontryagin's principle.

In the sequel, we assume that the set $U$ is given in the form

$$
U=\left\{u \in \mathbb{R}^{m} \mid g(u) \leq 0\right\},
$$

where $g: \mathbb{R}^{m} \rightarrow \mathbb{R}^{q}$ is a $C^{2}$ mapping. In other words, the control constraints are defined by

$$
g_{j}(u(t)) \leq 0, \quad \text { for a.a. } \quad t \in[0, T], \quad j=1, \ldots, q .
$$

We assume that the following qualification hypothesis of linear independence holds: the gradients $g_{i}^{\prime}(u), i \in I_{g}(u)$ are linearly independent at each point $u \in \mathbb{R}^{m}$ such that $g(u) \leq 0$, where $I_{g}(u)=\left\{i \in\{1, \ldots, q\} \mid g_{i}(u)=0\right\}$ is the set of active indices.

Let us recall the first order necessary condition of a weak minimum, which is a local minimum in $\mathcal{W}$. To this end, define the augmented Pontryagin function $\bar{H}: \mathbb{R}^{m} \times \mathbb{R}^{n} \times \mathbb{R}^{n *} \times \mathbb{R}^{q *} \rightarrow \mathbb{R}$ by

$$
\bar{H}(u, y, p, a)=H(u, y, p)+a g(u) .
$$

For $w=(u, y) \in F(P)$, denote by $\Lambda_{0}$ the set of all tuples $\lambda=(\mu, p, a) \in \mathbb{R}^{(r+1) *} \times W^{1, \infty}\left(0, T ; \mathbb{R}^{n *}\right) \times$ $L^{\infty}\left(0, T ; \mathbb{R}^{q *}\right)$ of Lagrange multipliers such that the following relations hold

$$
\begin{aligned}
& \mu_{i} \geq 0, i=0, \ldots, r_{1}, \mu_{i} \phi_{i}(\eta)=0, i=1, \ldots, r_{1},|\mu|=1, \\
& a(t) \geq 0, a(t) g(u(t))=0, \text { a.a. } t \in(0, T), \\
& -\dot{p}(t)=H_{y}(w(t), p(t)), \text { a.a. } t \in(0, T), \\
& p(0)=-\varphi_{y_{0}}^{\mu}(\eta), p(T)=\varphi_{y_{T}}^{\mu}(\eta), \\
& \bar{H}_{u}(w(t), p(t), a(t))=0, \text { a.a. } t \in(0, T),
\end{aligned}
$$

where $\eta=(y(0), y(T))$. The following result is well-known (see, e.g., [6], pp. 422-429, [13], pp. 148-149, and [17], p. 13).

Theorem 2.5. Let $w$ be a weak minimum. Then the set $\Lambda_{0}$ is nonempty and bounded. Moreover, the projector $(\mu, p, a) \rightarrow \mu$ is injective on $\Lambda_{0}$, and hence $\Lambda_{0}$ is a finite dimensional compact set.

Denote by $M_{0}$ the set of all $\lambda=(\mu, p, a) \in \Lambda_{0}$ such that inequality (2.10) of Pontryagin's principle is satisfied. Obviously, $M_{0} \subset \Lambda_{0}$, and the condition $M_{0} \neq \emptyset$ is equivalent to Pontryagin's principle.

\section{Growth CONDition of oRder $\gamma$}

Let us fix a pair $w=(u, y) \in F(P)$. By $\delta w=(\delta u, \delta y)$ we denote a variation, i.e., an arbitrary element of the space $\mathcal{W}$, and the notation $\left\{\delta w_{k}\right\}$ stands for an arbitrary sequence of variations in $\mathcal{W}$. For any $\delta w \in \mathcal{W}$ we set

$$
\delta f=f(u(t)+\delta u(t), y(t)+\delta y(t))-f(u(t), y(t))=f(w(t)+\delta w(t))-f(w(t)),
$$

i.e., $\delta f$ is the increment of the function $f$ (at the point $w$ ) which corresponds to the variation $\delta w$. Similarly, we set

$$
\delta \phi_{0}=\phi_{0}(y(0)+\delta y(0), y(T)+\delta y(T))-\phi_{0}(y(0), y(T))=\phi_{0}(\eta+\delta \eta)-\phi_{0}(\eta),
$$

etc. 
Definition 3.1. A function $\Gamma: \mathbb{R}^{m} \rightarrow \mathbb{R}$ is said to be an order function if there exists a number $\varepsilon_{\Gamma}>0$ such that:

(a) $\Gamma(u)=|u|^{2}$ if $|u|<\varepsilon_{\Gamma}$;

(b) $\Gamma(u)>0$ if $|u| \geq \varepsilon_{\Gamma}$;

(c) $\Gamma(u)$ is Lipschitz continuous on each compact set $\mathcal{C} \subset \mathbb{R}^{m}$.

Obviously, the function $\Gamma(u)=|u|^{2}$ is an order function. For an arbitrary order function $\Gamma(u)$, we set

$$
\gamma(\delta w)=\|\delta y\|_{\infty}^{2}+\int_{0}^{T} \Gamma(\delta u(t)) \mathrm{d} t .
$$

Following [8], p. 123, we call the functional $\gamma: \mathcal{W} \rightarrow \mathbb{R}$ the order (of minimum).

Further, following [8], p. 112, we define the violation function

$$
\sigma(\delta w)=\left(\delta \phi_{0}\right)_{+}+\sum_{i=1}^{r_{1}} \phi_{i}(\eta+\delta \eta)_{+}+\sum_{i=r_{1}+1}^{r}\left|\phi_{i}(\eta+\delta \eta)\right|+\|\delta \dot{y}-\delta f\|_{1},
$$

where $\eta=\left(y_{0}, y_{T}\right)=(y(0), y(T)), \delta \eta=\left(\delta y_{0}, \delta y_{T}\right)=(\delta y(0), \delta y(T)),\left(\delta \phi_{0}\right)_{+}=\left(\phi_{0}(\eta+\delta \eta)-\phi_{0}(\eta)\right)_{+}, \alpha_{+}=$ $\max \{\alpha, 0\}$.

Definition 3.2. We say that $\left\{\delta w_{k}\right\}$ is a bounded strong sequence if

$$
\sup _{k}\left\|\delta u_{k}\right\|_{\infty}<\infty, \quad\left|\delta y_{k}(0)\right|+\left\|\delta \underline{y}_{k}\right\|_{\infty} \rightarrow 0 \quad(k \rightarrow \infty) .
$$

Denote by $S$ the set of all bounded strong sequences satisfying:

$$
\begin{aligned}
& \text { (a) } u(t)+\delta u_{k}(t) \in U \text { for a.a. } t \in[0, T] \text { for all } k \text {; } \\
& \text { (b) } \sigma\left(\delta w_{k}\right) \rightarrow 0(k \rightarrow \infty) \text {. }
\end{aligned}
$$

Definition 3.3. We say that a bounded strong $\gamma$-sufficiency holds (at a point $w \in F(P)$ ) (cf. [8], p. 126) if there exists a constant $C>0$ such that for any sequence $\left\{\delta w_{k}\right\} \in S$ we have $\sigma\left(\delta w_{k}\right) \geq C \gamma\left(\delta w_{k}\right)$ for all sufficiently large $k$.

Equivalently, a bounded strong $\gamma$-sufficiency holds iff there exists $C>0$ such that for any $M>0$ there exists $\varepsilon>0$ such that the conditions

$$
\begin{aligned}
& u(t)+\delta u(t) \in U \text { for a.a. } t \in[0, T], \quad\|\delta u\|_{\infty}<M, \\
& \sigma(\delta w)<\varepsilon, \quad|\delta y(0)|<\varepsilon, \quad\|\underline{\delta y}\|_{\infty}<\varepsilon
\end{aligned}
$$

imply the inequality $\sigma(\delta w) \geq C \gamma(\delta w)$.

Definition 3.4. We say that a bounded strong $\gamma$-growth condition holds for the cost function $J$ (at a point $w \in F(P))$ if there exists $C>0$ such that, for any sequence $\left\{\delta w_{k}\right\} \in S$ satisfying the condition $w+\delta w_{k} \in F(P)$ for all $k$, we have $\delta_{k} J \geq C \gamma\left(\delta w_{k}\right)$ for all sufficiently large $k$, where $\delta_{k} J=J\left(\eta+\delta \eta_{k}\right)-J(\eta)$.

Equivalently, a bounded strong $\gamma$-growth condition holds for the cost function $J$ iff there exists $C>0$ such that for any $M>0$ there exists $\varepsilon>0$ such that the conditions $w+\delta w \in F(P),\|\delta u\|_{\infty}<M,\|\delta y\|_{\infty}<\varepsilon$, $|\delta y(0)|<\varepsilon$, and $\delta J<\varepsilon$ imply the inequality $\delta J \geq C \gamma(\delta w)$.

Theorem 3.5. For any order function $\Gamma(u)$ :

(a) a bounded strong $\gamma$-sufficiency implies a bounded strong $\gamma$-growth condition for the cost function; and

(b) a bounded strong $\gamma$-growth condition for the cost function implies a strict bounded strong minimum. 
Proof. (a) Assume that a bounded strong $\gamma$-sufficiency holds, i.e., there exists a constant $C>0$ such that for any sequence $\left\{\delta w_{k}\right\} \in S$ we have $\sigma\left(\delta w_{k}\right) \geq C \gamma\left(\delta w_{k}\right)$ for all sufficiently large $k$. Then for any sequence $\left\{\delta w_{k}\right\} \in S$ satisfying the condition $w+\delta w_{k} \in F(P)$ for all $k$, we have $\sigma\left(\delta w_{k}\right)=\left(\delta_{k} J\right)_{+} \geq C \gamma\left(\delta w_{k}\right)$ for all sufficiently large $k$. The latter means that the $\gamma$-growth condition for the cost function holds at $w$.

(b) Now assume that $w$ is not a point of a strict bounded strong minimum, i.e., there exists a sequence $w_{k} \in F(P)$, bounded in $\mathcal{W}$, such that $\underline{y}_{k} \rightarrow \underline{y}$ uniformly, $y_{k}(0) \rightarrow y(0), J\left(w_{k}\right) \leq J(w)$ and $w_{k} \neq w$ for all $k$. Set $\delta w_{k}=w_{k}-w$ for all $k$. Then, $\delta w_{k} \neq 0, \sigma\left(\delta w_{k}\right)=0, u(t)+\delta u_{k}(t) \in U$ for a.a. $t \in[0, T]$, for all $k$, and conditions (3.3) hold. Consequently, $\left\{\delta w_{k}\right\} \in S, \gamma\left(\delta w_{k}\right)>0$ and $\delta_{k} J \leq 0$ for all $k$. This implies that a bounded strong $\gamma$-growth condition for the cost function does not hold at $w$.

Corollary 3.6. For any order function $\Gamma(u)$, a bounded strong $\gamma$-sufficiency implies a strict bounded strong minimum.

Set $(c f .[8]$, p. 126)

$$
C_{\gamma}(\sigma, S):=\inf _{\left\{\delta w_{k}\right\} \in S}\left(\liminf _{k} \frac{\sigma\left(\delta w_{k}\right)}{\gamma\left(\delta w_{k}\right)}\right),
$$

where the lower bound is taken over the set of all sequences from $S$ that do not vanish. The following proposition easily follows from the definitions.

Proposition 3.7. The inequality $C_{\gamma}(\sigma, S)>0$ is equivalent to the bounded strong $\gamma$-sufficiency.

Our goal is to obtain conditions which guarantee the inequality $C_{\gamma}(\sigma, S)>0$. To this end, we will estimate $C_{\gamma}(\sigma, S)$ from below.

In order to formulate second order sufficient conditions for a bounded strong $\gamma$-sufficiency we shall need the following strengthening of condition (2.10) of the maximum principle.

Definition 3.8. Let $\lambda=(\mu, p, a) \in M_{0}$. We say that the function $H(v, y(t), p(t))$ satisfies the growth condition of the order $\Gamma$ if there exists $C>0$ such that

$$
\begin{aligned}
& H(v, y(t), p(t))-H(u(t), y(t), p(t)) \geq C \Gamma(v-u(t)) \\
& \text { for all } v \in U \text { and for a.a. } t \in[0, T] .
\end{aligned}
$$

For a given $C>0$, denote by $M(C \Gamma)$ the set of all $\lambda \in M_{0}$ such that condition (3.6) holds.

Remark 3.9. It was shown in [19], pp. 372-374, and [20], pp. 275-276, that a bounded strong $\gamma$-sufficiency implies the existence of $C>0$ such that $M(C \Gamma) \neq \emptyset$. The latter implies the continuity of the control $u(t)[2]$, Lemma 2.7. The case of discontinuous (piecewise continuous) control $u(t)$ corresponds to a certain finer order than (3.1). The no-gap conditions of this order were obtained in [19,20], and their proofs will be published elsewhere. The proofs of sufficient optimality conditions for a piecewise continuous control appeared in [22]. Also note that condition (3.6) is never fulfilled in the cases of singular or bang-bang controls, in problems linear in control. These cases were studied, for example, in [4,5,17]. Finally, note that in this paper we do not discuss an important question of a characterization of condition (3.6) in terms of (strengthened) Legendre-type conditions. This will be the subject of another paper.

Remark 3.10. From Theorem 2.5 it follows that $M(C \Gamma)$ is a finite dimensional compact set.

\section{SECOND ORDER SUfFICIENT CONDITIONS}

A direction (variation) $\delta w=(\delta u, \delta y) \in \mathcal{W}$ is said to be critical (cf. [6], Sect. 2) at a point $w \in F(P)$ if the following relations hold

$$
\begin{aligned}
& \phi_{i}^{\prime}(\eta) \delta \eta \leq 0, i \in I_{\phi}(\eta) \cup\{0\} ; \phi_{j}^{\prime}(\eta) \delta \eta=0, j=r_{1}+1, \ldots, r \\
& \delta \dot{y}=f^{\prime}(w) \delta w \\
& \left(g_{j}^{\prime}(u) \delta u\right) \chi_{\left\{g_{j}(u)=0\right\}} \leq 0, \text { a.a. } t \in[0, T], j=1, \ldots, q
\end{aligned}
$$


where $I_{\phi}(\eta)=\left\{i \in\left\{1, \ldots, r_{1}\right\} \mid \phi_{i}(\eta)=0\right\}$ is the set of active indices, $\chi_{\left\{g_{j}(u)=0\right\}}$ is the characteristic function of the set $\left\{t \in[0, T] \mid g_{j}(u(t))=0\right\}, j=1, \ldots, q, \eta=(y(0), y(T))$, and $\delta \eta=(\delta y(0), \delta y(T))$. Denote by $\mathcal{K}$ the set of all critical directions $\delta w \in \mathcal{W}$ at $w$. Obviously, $\mathcal{K}$ is a convex cone in $\mathcal{W}$. We call it the critical cone.

For any $\lambda=(\mu, p, a) \in \Lambda_{0}$, let us define a quadratic form (at $w$ ) by the relation

$$
\Omega(\delta w, \lambda):=\frac{1}{2}\left\langle\varphi_{\eta \eta}(\eta, \mu) \delta \eta, \delta \eta\right\rangle+\frac{1}{2} \int_{0}^{T}\left\langle\bar{H}_{w w}(w, p, a) \delta w, \delta w\right\rangle \mathrm{d} t
$$

where $\varphi_{\eta \eta}(\eta, \mu)$ is the second partial derivative (w.r.t. $\eta$ ) of the function $\varphi(\eta, \mu)$ defined by $(2.6)$ and $\bar{H}_{w w}(w, p, a)$ is the second partial derivative (w.r.t. $w)$ of the function $\bar{H}(w, p, a)$ defined by $(2.13)$. Obviously, $\Omega(\delta w, \lambda)$ is quadratic in $\delta w$ and linear in $\lambda$. For any $C \geq 0$ such that $M(C \Gamma) \neq \emptyset$, set

$$
\Omega_{M(C \Gamma)}(\delta w):=\max _{\lambda \in M(C \Gamma)} \Omega(\delta w, \lambda)
$$

In (4.5), the maximum exists, since $M(C \Gamma)$ is a finite dimensional compact set. Our main result is the following.

Theorem 4.1. Let $w=(u, y)$ be a feasible point of problem (2.1)-(2.5) (with the set $U$ defined by (2.11)), where $f: \mathbb{R}^{m} \times \mathbb{R}^{n} \rightarrow \mathbb{R}^{n}, \phi_{i}: \mathbb{R}^{n} \times \mathbb{R}^{n} \rightarrow \mathbb{R}(i=0, \ldots, r)$, and $g: \mathbb{R}^{m} \rightarrow \mathbb{R}^{q}$ are $C^{2}$ mappings. Assume that the mapping $g$ satisfies the qualification hypothesis of linear independence (see Sect. 2). Let there exist an order function $\Gamma$ and a number $C>0$ such that the set $M(C \Gamma)$ is nonempty and let there exist a number $C_{\mathcal{K}}>0$ such that

$$
\Omega_{M(C \Gamma)}(\delta w) \geq C_{\mathcal{K}}\left(|\delta y(0)|^{2}+\int_{0}^{T}|\delta u|^{2} \mathrm{~d} t\right) \quad \text { for all } \quad \delta w \in \mathcal{K} .
$$

Then, for the higher order $\gamma$ defined by (3.1), a bounded strong $\gamma$-sufficiency holds at $w$.

Remark 4.2. It was proved in [19], Chapter 5, and [20], Chapter 4, that if $w$ is a Pontryagin minimum, then the set $M_{0}$ is nonempty and $\max _{\lambda \in M_{0}} \Omega(\delta w, \lambda) \geq 0$ for all $\delta w \in \mathcal{K}$. Thus, the sufficient condition given by Theorem 4.1 is a natural strengthening of the necessary condition. Also, it was proved in [19], pp. 323-325, and [20], Supplement, pp. 154-156, that if, in the definition of critical cone $\mathcal{K}$, the condition $\delta w=(\delta u, \delta y) \in \mathcal{U} \times \mathcal{Y}$ is replaced by $\delta w=(\delta u, \delta y) \in \mathcal{U}_{2} \times \mathcal{Y}_{2}$, where $\mathcal{U}_{2}=L^{2}\left(0, T, \mathbb{R}^{m}\right)$ and $\mathcal{Y}_{2}=W^{1,2}\left(0, T, \mathbb{R}^{n}\right)$, the new condition of the form (4.6) is equivalent to the original one (this equivalence is not obvious).

Remark 4.3. In the literature, there are sufficient conditions for strong local optimality that require (i) one tuple of Lagrange multipliers at which the corresponding quadratic form is positive definite on a larger critical cone; (ii) the strengthened Legendre-Clebsch condition; (iii) the existence of a "unique" control maximizing the Hamiltonian (the strengthened condition of Pontryagin's principle) which means that $\operatorname{argmin}_{u} H(x(t), u, \psi(t))$ is a singleton equal to $\{u(t)\}$ a.a., and (iv) a certain growth condition (w.r.t. $u$ ) of the Hamiltonian at the infinity (cf., e.g., [21], Sect. 10.4, Thm. 10.5). Since (i) and (ii) form a pair of sufficient conditions for weak local optimality, (iii) and (iv) complement them to a set of conditions being sufficient for strong local optimality. Moreover, (i) can be equivalently represented in the form of the strengthened Jacobi-type condition ( $c f$. , e.g., [23]) or in the form of the existence of a solution to the corresponding Riccati equation (cf., e.g., [24]). Let us briefly compare Theorem 4.1 of the present paper with a known result by Vera Zeidan for strong local minimum given in [24], p. 1308, Theorem 6.1. In [24], the optimal control problem, referred as problem $(C)$, has the form: minimize $J(x, u):=l(x(b))+\int_{a}^{b} g(t, x(t) u(t)) \mathrm{d} t$ subject to $\dot{x}(t)=f(t, x(t), u(t)), x(a)=A$, $\psi(x(b))=0$, and $G_{i}(t, x(t), u(t)) \leq 0, i=1, \ldots, k$. So, in comparison with $(P)$, problem $(C)$ has mixed statecontrol inequality constraints (instead of pure control inequality constraints), but has no endpoint inequality constraints. Let $(\hat{x}, \hat{u})$ be an extremal such that $\hat{u} \in L^{\infty}$. It is assumed that the gradients $G_{i u}(t, x, u)$ of active constraints are uniformly linearly independent along the trajectory $(t, \hat{x}(t), \hat{u}(t))$ and moreover, there exists a tuple of Lagrange multipliers with $\lambda_{0} \neq 0$, where $\lambda_{0}$ is the Lagrange multiplier of $J$. Let $q_{i}(t)$ be the Lagrange multiplier of the constraint $G_{i}(t, x, u) \leq 0$. For any $\gamma \geq 0$, set $J_{\gamma}(t)=\left\{i \in\{1, \ldots, k\}: q_{i}(t)>\gamma\right\}$. In [24], 
it is assumed that there exists $\gamma>0$ such that $J_{\gamma}(t)=J_{0}(t)$ a.e. This is a very strong assumption removing certain difficulties in the proofs. Consider the case, where the control $\hat{u}(t)$ is continuous and $f, g$, and $G$ are independent of $t$. In this case, all $q_{i}(t)$ are continuous and the assumption $J_{\gamma}(t) \equiv J_{0}(t)(\gamma>0)$ means that for any $i=1, \ldots, k$ we have: (a) $q_{i}(t)>0$ for all $t \in[a, b]$ or (b) $q_{i}(t) \equiv 0$ on $[a, b]$. In [24], the critical cone (corresponding to the Riccati equation) is enlarged up to a subspace in such a way that among all $G_{i} \leq 0$ only the constraints of type (a) are taken into account in the sufficient conditions, while the constraints of type (b) are simply ignored. Therefore, in the case of continuous control, sufficient conditions of weak minimum in [24] turned out to be much stronger than those in the present paper. Now let us turn to sufficient conditions for strong minimum. We can prove that the strengthened Legendre-Clebsch condition (ii) together with the strengthened condition of Pontryagin's principle (iii) guarantee the existence of order function $\Gamma$ (Def. 3.1) such that the growth condition of order $\Gamma$ holds for the Hamiltonian (Def. 3.8). Hence (according to Thm. 4.1), (iii) complements (i) and (ii) to the set of sufficient conditions for bounded strong minimum. To guarantee a strong minimum, one may add some kind of growth condition (iv) for the Hamiltonian at the infinity (or assume that the inequality $g(u) \leq 0$ define a compact set in $\mathbb{R}^{m}$ ). In [24], the following condition complements (i) and (ii) to the set of sufficient conditions for strong minimum (and hence play the same role as pair (iii) and (iv)): there exists a mapping $\tilde{u}(t, x, p)$ (defined in some neighborhood of the trajectory $(\hat{x}(t), \hat{p}(t)$ ), where $\hat{p}(t)$ is the adjoint variable) such that (a) $\tilde{u}(t, \cdot, \cdot, \cdot)$ is continuous uniformly in $t$, (b) $\tilde{u}(t, \hat{x}(t), \hat{p}(t))=\hat{u}(t)$ a.e., and (c) $\tilde{u}(t, x, p) \in \operatorname{argmin}_{u}\{p f(t, x, u)+g(t, x, u): G(t, x, u) \leq 0\}$ (where $p$ corresponds to the minimum principle). Obviously, the latter condition strengthens the Pontryagin principle, but a detailed comparison of this condition with (iii) and (iv) is not so simple and could be done elsewhere.

The proof of Theorem 4.1 will require preparatory sections, namely, Sections 5-8. This proof will be completed in Section 9. The proofs of several important lemmas will be gathered in Section 10; one of these lemmas (namely, Lem. 7.2) will be based on the results of Section 11 concerning Hoffman's error bounds.

\section{Bounded strong And Pontryagin's SEquences}

Let us introduce a set of Pontryagin's sequences $\Pi$ related to the notion of Pontryagin minimum. In what follows, we omit the subscript $k$ in the notation of sequences.

Definition 5.1. We say that $\{\delta w\}$ is a Pontryagin's sequence if the following conditions are satisfied: $\limsup \|\delta u\|_{\infty}<\infty,\|\delta u\|_{1} \rightarrow 0$, and $\|\delta y\|_{1,1} \rightarrow 0$. Denote by $\Pi$ the set of all Pontryagin's sequences.

\subsection{Passage to Pontryagin's sequences, the basic constant}

Given any $\lambda=(\mu, p, a) \in \Lambda_{0}$ and $\delta w \in \mathcal{W}$, we define the following Lagrange function for the cost function (2.5) and constraints (2.1), (2.3) and (2.4):

$$
\Psi(\delta w, \lambda):=\delta \varphi^{\mu}-\int_{0}^{T} p(\delta \dot{y}-\delta f) \mathrm{d} t=\delta \varphi^{\mu}-\int_{0}^{T}(p \delta \dot{y}-\delta H) \mathrm{d} t
$$

where $\delta \varphi^{\mu}=\varphi^{\mu}(\eta+\delta \eta)-\varphi^{\mu}(\eta)$ (recall that $\varphi^{\mu}$ was defined in (2.6)) and $\delta H=p \delta f$. Below we shall need another representation of $\Psi$. Using (2.8), we get

$$
\begin{aligned}
\int_{0}^{T} p \delta \dot{y} \mathrm{~d} t & =\left.p \delta y\right|_{0} ^{T}-\int_{0}^{T} \dot{p} \delta y \mathrm{~d} t \\
& =\varphi_{y_{0}}^{\mu}(\eta) \delta y(0)+\varphi_{y_{T}}^{\mu}(\eta) \delta y(T)+\int_{0}^{T} H_{y}(w, p) \delta y \mathrm{~d} t
\end{aligned}
$$

for any $\lambda \in \Lambda_{0}$. Consequently,

$$
\Psi(\delta w, \lambda)=\delta \varphi^{\mu}-\varphi_{\eta}^{\mu}(\eta) \delta \eta+\int_{0}^{T}\left(\delta H-H_{y}(w, p) \delta y\right) \mathrm{d} t, \quad \forall \lambda \in \Lambda_{0} .
$$


Set

$$
\Psi_{\Lambda_{0}}(\delta w)=\max _{\lambda \in \Lambda_{0}} \Psi(\delta w, \lambda)
$$

Since $\Lambda_{0}$ is a bounded set, using (3.2) and (5.1) it is easy to show that there exists $k_{0}>0$ such that

$$
\Psi_{\Lambda_{0}}(\delta w) \leq k_{0} \sigma(\delta w) .
$$

In what follows we assume that, for a given order function $\Gamma$ and a number $C>0$, the set $M(C \Gamma)$ is nonempty. Using estimate (5.5), let us show that any sequence from $S$ is a Pontryagin's sequence.

Proposition 5.2. If $\{\delta w\} \in S$, then $\|\delta u\|_{1} \rightarrow 0,\|\delta y\|_{1,1} \rightarrow 0$, and $\gamma(\delta w) \rightarrow 0$.

The proof of this proposition will be based on two lemmas.

Lemma 5.3. If $\{\delta w\} \in S$ and $(\mu, p, a) \in \Lambda_{0}$, then $\left(\int_{0}^{T} \delta H \mathrm{~d} t\right)_{+} \rightarrow 0$.

Proof. According to (5.3)-(5.5), $\delta \varphi^{\mu}-\varphi_{\eta}^{\mu}(\eta) \delta \eta+\int_{0}^{T}\left(\delta H-H_{y}(w, p) \delta y\right) \mathrm{d} t \leq k_{0} \sigma(\delta w)$. Since $\|\delta y\|_{\infty} \rightarrow 0$, the condition $\sigma(\delta w) \rightarrow 0$ implies $\left(\int_{0}^{T} \delta H \mathrm{~d} t\right)_{+} \rightarrow 0$.

Lemma 5.4. If $\{\delta w\} \in S$, then $\int_{0}^{T} \Gamma(\delta u) \mathrm{d} t \rightarrow 0$ and $\int_{0}^{T}|\delta u|^{2} \mathrm{~d} t \rightarrow 0$.

Proof. Let $\{\delta w\} \in S$ and $(\mu, p, a) \in M(C \Gamma)(C>0)$. We have $\delta H:=H(w+\delta w, p)-H(w, p)=\bar{\delta}_{y} H+\delta_{u} H$, where $\bar{\delta}_{y} H=H(u+\delta u, y+\delta y, p)-H(u+\delta u, y, p), \delta_{u} H=H(u+\delta u, y, p)-H(w, p)$. The conditions $\|\delta \underline{y}\|_{\infty} \rightarrow 0$ and $\limsup \|\delta u\|_{\infty}<\infty$ imply that $\left\|\bar{\delta}_{y} H\right\|_{\infty} \rightarrow 0$. Therefore, the condition $\left(\int_{0}^{T} \delta H \mathrm{~d} t\right)_{+} \rightarrow 0$ (which holds by Lem. 5.3) implies $\left(\int_{0}^{T} \delta_{u} H \mathrm{~d} t\right)_{+} \rightarrow 0$. But $\delta_{u} H \geq C \Gamma(\delta u)$, since $(\mu, p, a) \in M(C \Gamma)$ and $u(t)+\delta u(t) \in U$ a.e. Consequently, $\int_{0}^{T} \Gamma(\delta u) \mathrm{d} t \rightarrow 0$. This implies that $\int_{0}^{T}|\delta u|^{2} \mathrm{~d} t \rightarrow 0$.

Proof of Proposition 5.2. Let $\{\delta w\} \in S$. Then by Cauchy-Schwartz inequality and by Lemma 5.4 we have $\|\delta u\|_{1} \leq \sqrt{T}\|\delta u\|_{2} \rightarrow 0$. Moreover, from conditions $\|\delta u\|_{1} \rightarrow 0,|\delta y(0)|+\|\delta \underline{y}\|_{\infty} \rightarrow 0$, and $\|\delta \dot{y}-\delta f\|_{1} \rightarrow 0$ we easily deduce that $\|\delta y\|_{1,1} \rightarrow 0$ and hence $\|\delta y\|_{\infty} \rightarrow 0$. Also, by Lemma $5.4, \int_{0}^{T} \Gamma(\delta u) \mathrm{d} t \rightarrow 0$. Consequently, $\gamma(\delta w) \rightarrow 0$.

Corollary 5.5. We have: $S \subset \Pi$ and moreover,

$$
S=\{\{\delta w\} \in \Pi \mid g(u(t)+\delta u(t)) \leq 0\},
$$

where the condition $g(u(t)+\delta u(t)) \leq 0$ is assumed to be satisfied for a.a. $t \in[0, T]$ and for all members of the sequence $\{\delta w\}$.

Proof. The inclusion $S \subset \Pi$ follows from Proposition 5.2. Further, for any $\{\delta w\} \in \Pi$, we obviously have $\sigma(\delta w) \rightarrow 0$. Therefore, $\{\{\delta w\} \in \Pi \mid g(u(t)+\delta u(t)) \leq 0\} \subset S$. This and the inclusion $S \subset \Pi$ implies equality (5.6), since any sequence from $S$ satisfies $g(u(t)+\delta u(t)) \leq 0$.

Set

$$
\Pi_{\sigma \gamma}=\{\{\delta w\} \in \Pi \mid g(u(t)+\delta u(t)) \leq 0, \sigma(\delta w) \leq O(\gamma(\delta w))\} .
$$

From equality (5.6) and definition (5.7) we easily deduce that

$$
C_{\gamma}(\sigma, S)=C_{\gamma}\left(\sigma, \Pi_{\sigma \gamma}\right)
$$

where the definition of $C_{\gamma}\left(\sigma, \Pi_{\sigma \gamma}\right)$ is similar to the definition (3.5) of $C_{\gamma}(\sigma, S)$. Using (5.5), we get

$$
C_{\gamma}\left(\sigma, \Pi_{\sigma \gamma}\right) \geq k_{0}^{-1} C_{\gamma}\left(\Psi_{\Lambda_{0}}, \Pi_{\sigma \gamma}\right)
$$


where, by definition

$$
C_{\gamma}\left(\Psi_{\Lambda_{0}}, \Pi_{\sigma \gamma}\right):=\inf _{\{\delta w\} \in \Pi_{\sigma \gamma}}\left(\liminf \frac{\Psi_{\Lambda_{0}}(\delta w)}{\gamma(\delta w)}\right)
$$

(the lower bound in this formula is taken over the set of sequences from $\Pi_{\sigma \gamma}$ that do not vanish). We call $C_{\gamma}:=C_{\gamma}\left(\Psi_{\Lambda_{0}}, \Pi_{\sigma \gamma}\right)$ the basic constant (on the set of Pontryagin's sequences). From (5.8) and (5.9) we obtain the following inequality

$$
C_{\gamma}(\sigma, S) \geq k_{0}^{-1} C_{\gamma}\left(\Psi_{\Lambda_{0}}, \Pi_{\sigma \gamma}\right) .
$$

In the sequel, we shall estimate the basic constant from below. Under conditions of Theorem 4.1, we shall show that this constant is positive. Then in view of (5.11), Theorem 4.1 will follow from Proposition 3.7.

\subsection{Extension of the set $\Pi_{\sigma \gamma}$}

In the sequel, we shall use one more representation for $\Psi$. Let $\lambda=(\mu, p, a) \in \Lambda_{0}$ and $\{\delta w\} \in \Pi$ satisfies the condition $u(t)+\delta u(t) \in U$ a.e. on $[0, T]$ for all members of the sequence. Then relation (5.3) combined with the equalities $a \delta g+a \delta g_{-}=0\left(\right.$ where $\alpha_{-}=\max \{-\alpha, 0\} \geq 0$ and $\left.g_{-}=\left(g_{1_{-}}, \ldots, g_{q_{-}}\right)\right)$and $\delta \bar{H}=\delta H+a \delta g$ implies

$$
\Psi(\delta w, \lambda)=\delta \varphi^{\mu}-\varphi_{\eta}^{\mu}(\eta) \delta \eta+\int_{0}^{T}\left(\delta \bar{H}-H_{y}(w, p) \delta y\right) \mathrm{d} t+\int_{0}^{T} a \delta g_{-} \mathrm{d} t .
$$

Assume in addition that $\{\delta w\} \in \Pi_{\sigma \gamma}$ and hence $\sigma(\delta w) \leq O(\gamma(\delta w)$ ). For given sequence, we deduce from (5.5) and (5.12) that

$$
\max _{\lambda \in \Lambda_{0}}\left\{\delta \varphi^{\mu}-\varphi_{\eta}^{\mu}(\eta) \delta \eta+\int_{0}^{T}\left(\delta \bar{H}-H_{y}(w, p) \delta y\right) \mathrm{d} t+\int_{0}^{T} a \delta g_{-} \mathrm{d} t\right\} \leq O(\gamma),
$$

where $\gamma:=\gamma(\delta w)$. Since $\bar{H}_{u}(w, p, a)=0, H_{y}=\bar{H}_{y}$ and limsup $\|\delta w\|_{\infty}<\infty$, the following estimate holds uniformly on $\Lambda_{0}: \int_{0}^{T}\left|\delta \bar{H}-H_{y}(w, p) \delta y\right| \mathrm{d} t \leq O(\gamma)$. Moreover, $\left|\delta \varphi^{\mu}-\varphi_{\eta}^{\mu}(\eta) \delta \eta\right| \leq O(\gamma)$ uniformly on $\Lambda_{0}$. Therefore, condition (5.13) implies

$$
\max _{\lambda \in \Lambda_{0}} \int_{0}^{T} a \delta g_{-} \mathrm{d} t \leq O(\gamma)
$$

This estimate is satisfied for any $\{\delta w\} \in \Pi_{\sigma \gamma}$. Hence we can rewrite (5.7) as

$$
\Pi_{\sigma \gamma}=\left\{\{\delta w\} \in \Pi \mid g(u+\delta u) \leq 0, \sigma \leq O(\gamma), \max _{\lambda \in \Lambda_{0}} \int_{0}^{T} a \delta g_{-} \mathrm{d} t \leq O(\gamma)\right\} .
$$

Since the set $\Lambda_{0}$ is finite dimensional, so is its convex hull co $\Lambda_{0}$. Hence the relative interior rico $\Lambda_{0}$ is nonempty. The following proposition will allow us to simplify the last relation in (5.15).

Proposition 5.6. Let $\hat{\lambda}=(\hat{\mu}, \hat{p}, \hat{a}) \in \operatorname{rico} \Lambda_{0}$. Then there exists $\hat{C}>0$ such that, for any $\lambda=(\mu, p, a) \in \operatorname{co} \Lambda_{0}$, the following inequalities hold

$$
\mu_{i} \leq \hat{C} \hat{\mu}_{i}, i=0, \ldots, r_{1} ; \quad a_{j}(t) \leq \hat{C} \hat{a}_{j}(t) \text { a.e. on }[0, T], j=1, \ldots, q .
$$

Proof. Since $\hat{\lambda}=(\hat{\mu}, \hat{p}, \hat{a})$ is an interior point of the bounded set co $\Lambda_{0}$, there exists $\rho>0$ such that for any $\lambda=(\mu, p, a) \in \operatorname{co} \Lambda_{0}$ we have $\hat{\lambda} \pm \rho(\lambda-\hat{\lambda}) \in \operatorname{co} \Lambda_{0}$. Condition $\hat{\lambda}-\rho(\lambda-\hat{\lambda}) \in \operatorname{co} \Lambda_{0}$ implies $\hat{\mu}_{i}-\rho\left(\mu_{i}-\hat{\mu}_{i}\right) \geq 0$, $i=0, \ldots, r_{1}$, and $\hat{a}_{j}(t)-\rho\left(a_{j}(t)-\hat{a}_{j}(t)\right) \geq 0, j=1, \ldots, q$. Consequently,

$$
\frac{1+\rho}{\rho} \hat{\mu}_{i} \geq \mu_{i}, \quad i=0, \ldots, r_{1} ; \quad \frac{1+\rho}{\rho} \hat{a}_{j}(t) \geq a_{j}(t), \quad j=1, \ldots, q .
$$

Thus, it suffices to set $\hat{C}=(1+\rho) / \rho$. 
Let us fix an element $\hat{\lambda}=(\hat{\mu}, \hat{p}, \hat{a}) \in \operatorname{rico} \Lambda_{0}$. It follows from Proposition 5.6, that condition (5.14) is equivalent to the condition $\int_{0}^{T} \hat{a}(\delta g)_{-} \mathrm{d} t \leq O(\gamma)$. Then, from (5.15) it follows that

$$
\Pi_{\sigma \gamma}=\left\{\{\delta w\} \in \Pi \mid g(u+\delta u) \leq 0, \sigma \leq O(\gamma), \int_{0}^{T} \hat{a} \delta g_{-} \mathrm{d} t \leq O(\gamma)\right\} .
$$

Since we estimate the basic constant from bellow, we can extend the set of sequences $\Pi_{\sigma \gamma}$. Namely, let us define a set of sequences

$$
\Pi_{o(\sqrt{\gamma})}=\left\{\{\delta w\} \in \Pi \mid g(u+\delta u) \leq 0, \sigma \leq o(\sqrt{\gamma}), \int_{0}^{T} \hat{a} \delta g_{-} \mathrm{d} t \leq O(\gamma)\right\} .
$$

Obviously, $\Pi_{\sigma \gamma} \subset \Pi_{o(\sqrt{\gamma})}$, and hence

$$
C_{\gamma}\left(\Psi_{\Lambda_{0}}, \Pi_{\sigma \gamma}\right) \geq C_{\gamma}\left(\Psi_{\Lambda_{0}}, \Pi_{o(\sqrt{\gamma})}\right),
$$

where the r.h.s. of inequality (5.19) is defined similarly to the l.h.s. of this inequality. In what follows, we will estimate $C_{\gamma}\left(\Psi_{\Lambda_{0}}, \Pi_{o(\sqrt{\gamma})}\right)$ from below.

\section{LOCAL SEQUENCES}

Our final goal is to obtain a lower bound of $C_{\gamma}$ defined by the set of sequences of critical variations (satisfying (4.1)-(4.3)) and by the quadratic form $\Omega$ (defined in (4.4)). It will be done in several steps. In each step lower bound of $C_{\gamma}$ is defined on a different set of sequences and a modification of the function $\Psi$ is used. In this section, we shall make one more step in this direction, namely, in the definition of $C_{\gamma}\left(\Psi_{\Lambda_{0}}, \Pi_{o(\sqrt{\gamma})}\right)$ we shall replace Pontryagin's sequences by the sequences satisfying the condition $\|\delta w\|_{\mathcal{W}} \rightarrow 0$. After that the obtained lower bound of $C_{\gamma}$ will be simplified.

\subsection{Passage to the sequences of local variations}

Set

$$
S^{\mathrm{loc}}=\left\{\{\delta w\} \mid\|\delta w\|_{\mathcal{W}}:=\|\delta u\|_{\infty}+\|\delta y\|_{1,1} \rightarrow 0\right\} .
$$

Sequences from $S^{\text {loc }}$ will be called local. Note that $S^{\text {loc }} \subset \Pi$. Bellow, we shall pass to the set of sequences

$$
S_{o(\sqrt{\gamma})}^{\mathrm{loc}}:=\Pi_{o(\sqrt{\gamma})} \cap S^{\mathrm{loc}} .
$$

In other words, $S_{o(\sqrt{\gamma})}^{\text {loc }}$ is the set of the sequences $\{\delta w\}$ satisfying the relations

$$
\begin{aligned}
& \|\delta w\|_{\mathcal{W}} \rightarrow 0, g(u+\delta u) \leq 0, \\
& \sigma=o(\sqrt{\gamma}), \int_{0}^{T} \hat{a}(\delta g)_{-} \mathrm{d} t \leq O(\gamma) .
\end{aligned}
$$

By (3.2), the relation $\sigma=o(\sqrt{\gamma})$ is equivalent to the set of relations

$$
\begin{aligned}
& \left\|\delta \dot{y}-f^{\prime}(w) \delta w\right\|_{1}=o(\sqrt{\gamma}), \\
& \phi_{i}^{\prime}(\eta) \delta \eta \leq o(\sqrt{\gamma}), i \in I_{\phi}(\eta) \cup\{0\}, \\
& \left|\phi_{i}^{\prime}(\eta) \delta \eta\right|=o(\sqrt{\gamma}), i=r_{1}+1, \ldots, r .
\end{aligned}
$$


The second relation in (6.4) is equivalent to

$$
\int_{0}^{T} \hat{a}_{j}\left(g_{j}^{\prime}(u) \delta u\right)_{-} \mathrm{d} t \leq O(\gamma), j=1, \ldots, q .
$$

Thus, the set $S_{o(\sqrt{\gamma})}^{\mathrm{loc}}$ can be characterizes by (6.3) and (6.5)-(6.8).

Lemma 6.1. For any $\lambda=(\mu, p, a) \in \Lambda_{0}$ and for any sequence $\{\delta w\} \in S^{\text {loc }}$ satisfying the relation $g(u+\delta u) \leq 0$ (for all members of the sequence) the following formula holds:

$$
\Psi(\delta w, \lambda)=\Omega(\delta w, \lambda)+\int_{0}^{T} a \delta g_{-} \mathrm{d} t+o(\gamma(\delta w))
$$

uniformly on $\Lambda_{0}$, where $\Omega$ is defined by (4.4).

Proof. Formula (6.9) follows from (5.12) and the relations: $H_{y}=\bar{H}_{y}$ and $\bar{H}_{u}(w, p, a)=0$ for all $\lambda \in \Lambda_{0}$.

For any $C>0$ such that the set $M(C \Gamma)$ is nonempty, we set

$$
\Psi_{M(C \Gamma)}(\delta w):=\max _{\lambda \in M(C \Gamma)} \Psi(\delta w, \lambda)
$$

Lemma 6.2. For any $C>0$ such that the set $M(C \Gamma)$ is nonempty, the following inequality holds

$$
C_{\gamma}\left(\Psi_{\Lambda_{0}}, \Pi_{o(\sqrt{\gamma})}\right) \geq \min \left\{C_{\gamma}\left(\Psi_{M(C \Gamma)}, S_{o(\sqrt{\gamma})}^{\mathrm{loc}}\right), C\right\}
$$

where the constant $C_{\gamma}\left(\Psi_{M(C \Gamma)}, S_{o(\sqrt{\gamma})}^{\text {loc }}\right)$ is defined as in (5.10).

The proof is given in Section 10.1 .

Recall that we have fixed $C>0$ such that the set $M(C \Gamma)$ is nonempty. Lemma 6.2 along with inequality (5.19) implies

$$
C_{\gamma}\left(\Psi_{\Lambda_{0}}, \Pi_{\sigma \gamma}\right) \geq \min \left\{C_{\gamma}\left(\Psi_{M(C \Gamma)}, S_{o(\sqrt{\gamma})}^{\mathrm{loc}}\right), C\right\}
$$

In what follows, we shall estimate $C_{\gamma}\left(\Psi_{M(C \Gamma)}, S_{o(\sqrt{\gamma})}^{\text {loc }}\right)$ from below.

\subsection{Passage to the set of sequences $S_{1}$}

Proposition 6.3. Let $\lambda=(\mu, p, a) \in \Lambda_{0}$. Then the critical cone $\mathcal{K}$, defined by (4.1)-(4.3), has the following equivalent representation:

$$
\begin{aligned}
& \phi_{i}^{\prime}(\eta) \delta \eta \leq 0, \mu_{i} \phi_{i}^{\prime}(\eta) \delta \eta=0, i \in I_{\phi}(\eta) \cup\{0\}, \\
& \phi_{j}^{\prime}(\eta) \delta \eta=0, j=r_{1}+1, \ldots, r, \delta \dot{y}=f^{\prime}(w) \delta w, \\
& \left(g_{j}^{\prime}(u) \delta u\right) \chi_{\left\{g_{j}(u)=0\right\}} \leq 0, a_{j} g_{j u}(u) \delta u=0, j=1, \ldots, q .
\end{aligned}
$$

Proof. Indeed, from definition (2.14) of the set $\Lambda_{0}$ it easily follows that, for any $\lambda=(\mu, p, a) \in \Lambda_{0}$ and any $\delta w \in \mathcal{W}$, we have

$$
\sum_{i=0}^{r} \mu_{i} \phi_{i}^{\prime}(\eta) \delta \eta-\int_{0}^{T} p\left(\delta \dot{y}-f^{\prime}(w) \delta w\right) \mathrm{d} t+\int_{0}^{T} a g^{\prime}(u) \delta u \mathrm{~d} t=0 .
$$

Therefore, relations (4.1)-(4.3) imply that $\mu_{i} \phi_{i}^{\prime}(\eta) \delta \eta=0, i \in I_{\phi}(\eta) \cup\{0\}, a g^{\prime}(u) \delta u=0$. Thus, relations (6.13) follows from (4.1)-(4.3). Vice versa, relations (4.1)-(4.3) obviously follows from (6.13). 
Define a new set $S_{1}$ of sequences $\{\delta w\} \subset \mathcal{W}$ by the relations

$$
\begin{aligned}
& \|\delta u\|_{\infty}+\|\delta y\|_{1,1} \rightarrow 0, \sigma(\delta w)=o(\sqrt{\gamma(\delta w)}), \\
& g(u)+g^{\prime}(u) \delta u \leq 0, g_{j}^{\prime}(u) \delta u \chi_{\left\{\hat{a}_{j} \geq \varepsilon\right\}}=0, j=1, \ldots, q, \varepsilon \rightarrow+0,
\end{aligned}
$$

where $\chi_{\left\{\hat{a}_{i} \geq \varepsilon\right\}}(t)$ is the characteristic function of the set $\left\{t \mid \hat{a}_{i}(t) \geq \varepsilon\right\}$. The equalities in (6.15) mean that for a given sequence $\left\{\delta w_{k}\right\}$ there exists a sequence $\left\{\varepsilon_{k}\right\}$ such that $\varepsilon_{k}>0, \varepsilon_{k} \rightarrow 0$, and $g_{j}^{\prime}(u(t)) \delta u_{k}(t)=0$ a.e. on the set $\left\{t \mid \hat{a}_{j}(t) \geq \varepsilon_{k}\right\}$ for all $j=1, \ldots, q$ and for all $k=1,2, \ldots$ Set

$$
\Phi_{C}^{1}(\delta w):=\max _{\lambda \in M(C \Gamma)}\left\{\Omega(\delta w, \lambda)+\int_{0}^{T} a\left(g^{\prime}(u) \delta u\right)_{-} \mathrm{d} t\right\} .
$$

Lemma 6.4. The following inequality holds

$$
C_{\gamma}\left(\Psi_{M(C \Gamma)}, S_{o(\sqrt{\gamma})}^{\mathrm{loc}}\right) \geq C_{\gamma}\left(\Phi_{C}^{1}, S_{1}\right)
$$

where

$$
C_{\gamma}\left(\Phi_{C}^{1}, S_{1}\right):=\inf _{\{\delta w\} \in S_{1}}\left(\liminf \frac{\Phi_{C}^{1}(\delta w)}{\gamma(\delta w)}\right) .
$$

The proof is given in Section 10.2.

\section{Support of the CRitical CONE}

Here we define a notion of support of the critical cone and formulate an important property of the support (Lem. 7.2) which will play a crucial role at the end of the proof of Theorem 4.1 in Section 9. This property will mean that the first order approximations of the endpoint functionals possess the so-called "Hoffman's error bound" [7] on the support.

Set

$$
\mathcal{W}_{0}=\left\{\delta w=(\delta u, \delta y) \in \mathcal{W} \mid \delta \dot{y}=f_{w}(w) \delta w\right\} .
$$

Consider two sets of linear functionals

$$
\begin{array}{ll}
l_{i}: \delta w \in \mathcal{W}_{0} \rightarrow \phi_{i}^{\prime}(\eta) \delta \eta, & i \in I_{\phi}(\eta) \cup\{0\}, \\
l_{i}: \delta w \in \mathcal{W}_{0} \rightarrow \phi_{i}^{\prime}(\eta) \delta \eta, & i=r_{1}+1, \ldots, r,
\end{array}
$$

where $\delta w=(\delta u, \delta y), \delta \eta=(\delta y(0), \delta y(T))$. Let $Q_{0}$ be the cone generated by functionals (7.2), and let $Q_{1}$ be the subspace generated by functionals (7.3). Set

$$
Q:=Q_{0}+Q_{1}
$$

Then $Q$ is a convex and finitely generated cone. Note that $w^{*} \in Q$ iff there is a vector $\mu=\left(\mu_{0}, \ldots, \mu_{r}\right) \in \mathbb{R}^{(r+1) *}$ such that $\mu_{i} \geq 0, i=0, \ldots, r_{1}, \mu_{i} \phi_{i}(\eta)=0, i=1, \ldots, r_{1}$ and $\left\langle w^{*}, \delta w\right\rangle=\varphi_{\eta}^{\mu}(\eta) \delta \eta$ for all $\delta w \in \mathcal{W}_{0}$, where $\varphi^{\mu}=\sum_{i=0}^{r} \mu_{i} \phi_{i}$, as in (2.6). Obviously, $\left\langle w^{*}, \delta w\right\rangle \leq 0$ for any $w^{*} \in Q$ and any $\delta w \in \mathcal{K}$. Moreover, let $p=p^{\mu}$ be the solution to the adjoint equation $-\dot{p}=p f_{y}(w), p(T)=\varphi_{y_{T}}^{\mu}(\eta)$. As it is well-known, the functional $\varphi_{\eta}^{\mu}(\eta) \delta \eta$ has the following representation on the subspace $\mathcal{W}_{0}:\left(\varphi_{y_{0}}^{\mu}(\eta)+p(0)\right) \delta y_{0}+\int_{0}^{T} p f_{u}(w) \delta u \mathrm{~d} t$. This representation is "pure integral" iff $\varphi_{y_{0}}^{\mu}(\eta)+p(0)=0$. 
Let us define the set

$$
\begin{aligned}
\mathcal{R}_{0}=\left\{\delta w=(\delta u, \delta y) \in \mathcal{W}_{0} \mid\right. & g_{j}^{\prime}(u) \delta u \chi_{\left\{g_{j}(u)=0\right\}} \leq 0, \\
& \left.\hat{a}_{j} g_{j}^{\prime}(u) \delta u=0, j=1, \ldots, q\right\},
\end{aligned}
$$

where $\hat{a}$ is given in Proposition 5.6. Obviously, $\mathcal{R}_{0}$ is a closed convex cone, and by Proposition 6.3, $\mathcal{K} \subset \mathcal{R}_{0}$. Let $w_{1}^{*} \in Q$ be a linear functional which has the following integral representation on the cone $\mathcal{R}_{0}:\left\langle w_{1}^{*}, \delta w\right\rangle=$ $-\int_{0}^{T} a^{1} g^{\prime}(u) \delta u \mathrm{~d} t \forall \delta w \in \mathcal{R}_{0}$, where $a^{1} \in L^{1}\left(0, T ; \mathbb{R}^{q *}\right), a^{1} \geq 0$, and $a^{1} g(u)=0$. Clearly, $\left\langle w_{1}^{*}, \delta w\right\rangle \geq 0$ for all $\delta w \in \mathcal{R}_{0}$, i.e. $w_{1}^{*} \in \mathcal{R}_{0}^{*}$. Assume that there exists $\delta w_{1} \in \mathcal{R}_{0}$ such that $\left\langle w_{1}^{*}, \delta w_{1}\right\rangle>0$. Set $\mathcal{R}_{1}=\left\{\delta w \in \mathcal{R}_{0} \mid\right.$ $\left.\left\langle w_{1}^{*}, \delta w\right\rangle=0\right\}$. Conditions (7.5) along with $a^{1} \geq 0$ and $a^{1} g(u)=0$ imply $\mathcal{R}_{1}=\left\{\delta w \in \mathcal{R}_{0} \mid a^{1} g^{\prime}(u) \delta u=0\right\}$. Moreover, since $w_{1}^{*} \in Q$, we have $\left\langle w_{1}^{*}, \delta w\right\rangle \leq 0$ for all $\delta w \in \mathcal{K}$. But $\mathcal{K} \subset \mathcal{R}_{0}$ and $w_{1}^{*} \in \mathcal{R}_{0}^{*}$, consequently $\left\langle w_{1}^{*}, \delta w\right\rangle=0$ for all $\delta w \in \mathcal{K}$. This implies that $\mathcal{K} \subset \mathcal{R}_{1}$.

Similarly, let $w_{2}^{*} \in Q$ be a linear functional which has an integral representation on the cone $\mathcal{R}_{1}:\left\langle w_{2}^{*}, \delta w\right\rangle=$ $-\int_{0}^{T} a^{2} g^{\prime}(u) \delta u \mathrm{~d} t \forall \delta w \in \mathcal{R}_{1}$, where $a^{2} \in L^{1}\left(0, T, \mathbb{R}^{q *}\right), a^{2} \geq 0, a^{2} g(u)=0$. Obviously, $\left\langle w_{2}^{*}, \delta w\right\rangle \geq 0 \forall \delta w \in \mathcal{R}_{1}$, i.e. $w_{2}^{*} \in \mathcal{R}_{1}^{*}$. Assume that there exists $\delta w_{2} \in \mathcal{R}_{1}$ such that $\left\langle w_{2}^{*}, \delta w_{2}\right\rangle>0$. Set $\mathcal{R}_{2}=\left\{\delta w \in \mathcal{R}_{1} \mid\left\langle w_{2}^{*}, \delta w\right\rangle=0\right\}$. Then $\mathcal{R}_{2}=\left\{\delta w \in \mathcal{R}_{1} \mid a^{2} g^{\prime}(u) \delta u=0\right\}$. From conditions $w_{2}^{*} \in Q, w_{2}^{*} \in \mathcal{R}_{1}^{*}$, and $\mathcal{K} \subset \mathcal{R}_{1}$ we deduce that $\left\langle w_{2}^{*}, \delta w\right\rangle=0 \forall \delta w \in \mathcal{K}$. Hence $\mathcal{K} \subset \mathcal{R}_{2}$.

Continuing this process, we obtain a set of functionals $w_{1}^{*}, w_{2}^{*}, \ldots, w_{s}^{*}$ and a set of cones $\mathcal{R}_{0}, \mathcal{R}_{1}, \mathcal{R}_{2}, \ldots, \mathcal{R}_{s}$ such that $\mathcal{K} \subset \mathcal{R}_{s} \subset \ldots \subset \mathcal{R}_{1} \subset \mathcal{R}_{0}$ and $w_{k}^{*}\left(\mathcal{R}_{k}\right)=\{0\}, w_{k}^{*}\left(\mathcal{R}_{k-1}\right) \neq\{0\}, k=1, \ldots, s$. Due to these properties, the functionals $w_{1}^{*}, w_{2}^{*}, \ldots, w_{s}^{*}$ are linearly independent. Since the cone $Q$ (containing these functionals) is finite generated, this process will be finished on some finite step $s$. Set $\mathcal{S}=\mathcal{R}_{s}, a^{0}=\hat{a}$. Thus

$$
\mathcal{S}=\left\{\delta w \in \mathcal{W}_{0} \mid \delta u \in \mathcal{S}_{u}\right\}
$$

where

$$
\begin{gathered}
\mathcal{S}_{u}:=\left\{\delta u \in \mathcal{U} \mid\left(g_{j}^{\prime}(u) \delta u\right) \chi_{\left\{g_{j}(u)=0\right\}} \leq 0, j=1, \ldots, q,\right. \\
\left.a^{i} g^{\prime}(u) \delta u=0, i=0,1, \ldots, s\right\} .
\end{gathered}
$$

We call $\mathcal{S}$ the support of the critical cone $\mathcal{K}$.

Remark 7.1. Without loss of generality we can assume that $a^{i} \in L^{\infty}\left(0, T ; \mathbb{R}^{m}\right), i=0,1, \ldots, s$. Indeed, for $a^{0}=\hat{a}$ this condition holds. Further, we can choose $a^{1}$ so that $a^{1} \geq 0, a^{1} g(u)=0$, and $a_{j}^{1}(t)=0$ a.e. on the set $\left\{t \mid a_{j}^{0}(t)>0\right\}, j=1, \ldots, q$. Also we have $\left\langle w_{1}^{*}, \delta w\right\rangle:=\varphi_{\eta}^{\mu^{1}}(\eta) \delta \eta=\int_{0}^{T} p^{1} f_{u}(w) \delta u \mathrm{~d} t=-\int_{0}^{T} a^{1} g^{\prime}(u) \delta u \mathrm{~d} t$ for all $\delta w \in \mathcal{R}_{0}$, where $p^{1}$ is the solution to the adjoint equation $-\dot{p}^{1}=p^{1} f_{y}(w), p^{1}(T)=\varphi_{y_{T}}^{\mu^{1}}(\eta)$. Using these conditions and the linear independence hypothesis for the gradients $g_{j u}$, we easily deduce that $a^{1} \in$ $L^{\infty}\left(0, T ; \mathbb{R}^{m}\right)$. Similarly, we can choose $a^{i} \in L^{\infty}\left(0, T ; \mathbb{R}^{m}\right)$ for $i>1$.

The following important lemma will be used in Section 9.

Lemma 7.2. There exists $N>0$ such that for any $\delta w=(\delta u, \delta y) \in \mathcal{S}$ there exists $\bar{w}=(\bar{u}, \bar{y}) \in \mathcal{S}$ such that the following relations hold

$$
\begin{array}{r}
\phi_{i}^{\prime}(\eta)(\delta \eta+\bar{\eta}) \leq 0, i \in I_{\phi} \cup\{0\}, \phi_{j}^{\prime}(\eta)(\delta \eta+\bar{\eta})=0, j=r_{1}+1, \ldots, r, \\
\|\bar{w}\|_{\mathcal{W}} \leq N\left(\sum_{i \in I_{\phi} \cup\{0\}}\left(\phi_{i}^{\prime}(\eta) \delta \eta\right)_{+}+\sum_{j=r_{1}+1}^{r}\left|\phi_{j}^{\prime}(\eta) \delta \eta\right|\right),
\end{array}
$$

where $\delta \eta=(\delta y(0), \delta y(T)), \bar{\eta}=(\bar{y}(0), \bar{y}(T))$.

The proof is given in Section 10.3. 


\section{Auxiliary ASSERTIONS}

Along with Lemma 7.2, the following three lemmas will be used in Section 9. Let $L_{+}^{p}(0, T ; \mathbb{R})$ denote the cone of nonnegative functions from $L^{p}(0, T ; \mathbb{R})(1 \leq p \leq \infty)$, and let $\mathbb{R}_{+}$be the set of nonnegative numbers.

Lemma 8.1. Let $r \in L_{+}^{\infty}(0, T, \mathbb{R})$, set $\mathcal{M}=\{t \mid r(t)>0\}$. Let $\{(v, \alpha)\}$ be a sequence in $L_{+}^{2}(0, T ; \mathbb{R}) \times \mathbb{R}_{+}$ such that $\alpha \rightarrow 0,\|v\|_{2} \leq \alpha$, and $\int_{0}^{T} r v \mathrm{~d} t=o(\alpha)$. Then there exists a sequence $\{B\}$ of subsets $B \subset \mathcal{M}$ such that meas $B \rightarrow 0$ and for the sequence of sets $\{A\}:=\{\mathcal{M} \backslash B\}$ we have $\left\|v \chi_{A}\right\|_{\infty}=o(\alpha)$, where $\chi_{A}$ is the characteristic function of the set $A$.

The proof is given in Section 10.4.

Denote by $S^{\prime}$ the set of sequences $\left\{u^{\prime}\right\}$ in $\mathcal{U}$ satisfying the relations

$$
\begin{aligned}
& \left\|u^{\prime}\right\|_{\infty} \rightarrow 0, \quad \operatorname{meas}\left\{t \mid u^{\prime}(t) \neq 0\right\} \rightarrow 0, \quad g(u)+g^{\prime}(u) u^{\prime} \leq 0, \\
& g_{j}^{\prime}(u) u^{\prime} \chi_{\left\{\hat{a}_{j} \geq \varepsilon\right\}}=0, j=1, \ldots, q, \quad \varepsilon \rightarrow+0 .
\end{aligned}
$$

The equalities in (8.1) mean that for the sequence $\left\{u_{k}^{\prime}\right\}$ there exists a sequence $\varepsilon_{k} \rightarrow+0$ such that $g_{j}^{\prime}(u(t)) u_{k}^{\prime}(t)=0$ a.e. on the set $\left\{t \mid \hat{a}_{j}(t) \geq \varepsilon_{k}\right\}, j=1, \ldots, q, k=1,2, \ldots$

Recall that by the assumption $M(C \Gamma) \neq \emptyset$, where $C>0$. The following assertion holds.

Lemma 8.2. For any $\left\{u^{\prime}\right\} \in S^{\prime}, C^{\prime}<C$, and $\lambda \in M(C \Gamma)$ we have

$$
\int_{0}^{T}\left(\left\langle\bar{H}_{u u}(w, p, a) u^{\prime}, u^{\prime}\right\rangle+a\left(g^{\prime}(u) u^{\prime}\right)_{-}\right) \mathrm{d} t \geq C^{\prime} \int_{0}^{T}\left|u^{\prime}\right|^{2} \mathrm{~d} t,
$$

starting from a certain member of the sequence $\left\{u^{\prime}\right\}$.

The proof is given in Section 10.5.

Recall that $S_{1}$ was defined in Section 6.2 as the set of sequences $\{\delta w\}$ in $\mathcal{W}$ satisfying relations (6.14) and (6.15). Equivalently, $S_{1}$ is the set of sequences such that $\|\delta w\|_{\mathcal{W}} \rightarrow 0$ and relations (6.15) and (6.5)-(6.7) hold. For the set of functionals $w_{1}^{*}, \ldots, w_{s}^{*}$, introduced in Section 7 , and for any $\{\delta w\} \in S_{1}$ relations (6.6) and (6.7) imply the upper bounds $\left\langle w_{i}^{*}, \delta w\right\rangle \leq o(\sqrt{\gamma}), i=1, \ldots, s$. This easily follows from the fact that each functional $\left\langle w_{i}^{*}, \delta w\right\rangle$ belongs to the set $Q$ (defined by (7.4)) and therefore has the form $\sum_{i \in I_{\phi} \cup\{0\}} \mu_{i} \phi_{i}^{\prime}(\eta) \delta \eta+$ $\sum_{i=r_{1}+1}^{r} \mu_{i} \phi_{i}^{\prime}(\eta) \delta \eta$, where $\mu_{i} \geq 0$ for all $i \in I_{\phi} \cup\{0\}$. Further, recall that each functional $w_{i}^{*}$ has an integral representation $\left\langle w_{i}^{*}, \delta w\right\rangle=-\int_{0}^{T} a^{i} g^{\prime}(u) \delta u \mathrm{~d} t$ on the cone $\mathcal{R}_{i-1}, i=1, \ldots, s$, where $a^{i} \in L^{\infty}\left(0, T ; \mathbb{R}^{m}\right), a^{i} \geq 0$, $a^{i} g(u)=0, i=1, \ldots, s$ (see Rem. 7.1).

Lemma 8.3. For any sequence $\{\delta w\} \in S_{1}$ we have

$$
-\int_{0}^{T} a^{i} g^{\prime}(u) \delta u \mathrm{~d} t \leq o(\sqrt{\gamma(\delta w)}), \quad i=1, \ldots, s .
$$

The proof is given in Section 10.6.

Remark 8.4. Although the upper bound $\left\langle w_{i}^{*}, \delta w\right\rangle \leq o(\sqrt{\gamma})$ holds for any $\{\delta w\} \in S_{1}$ and any $i=1, \ldots, s$, it does not automatically imply upper bound (8.3), since generally speaking elements of $\{\delta w\} \in S_{1}$ do not belong to the cone $\mathcal{R}_{i-1}$ on which $w_{i}^{*}$ has the representation $\left\langle w_{i}^{*}, \delta w\right\rangle=-\int_{0}^{T} a^{i} g^{\prime}(u) \delta u \mathrm{~d} t$.

\section{Proof of TheOrem 4.1}

We are going to estimate $C_{\gamma}\left(\Phi_{C}^{1}, S_{1}\right)$ (given in (6.18)) from below. To this end, choose any sequence $\{\delta w\} \in S_{1}$ that does not vanish. Using Lemmas 8.1 and 8.3 let us construct sequences $\left\{u^{\prime}\right\} \in S^{\prime}$ (where $S^{\prime}$ is given in (8.1)) 
and $\left\{\delta u_{1}\right\} \subset \mathcal{S}_{u}$ (where $\mathcal{S}_{u}$ is given in (7.7)) as follows. Set $v_{j}:=-g_{j}^{\prime}(u) \delta u \chi_{\left\{g_{j}(u)=0\right\}}, j=1, \ldots, q$. Then $v_{j} \geq 0$ and $\left\|v_{j}\right\|_{2} \leq O(\sqrt{\gamma}), j=1, \ldots, q$. By Lemma $8.3 \int_{0}^{T} a_{j}^{i} v_{j} \mathrm{~d} t \leq o(\sqrt{\gamma}), i=1, \ldots, s, j=1, \ldots, q$, where $\gamma=\gamma(\delta w)$. Set

$$
\mathcal{M}_{j}^{i}=\left\{t \mid a_{j}^{i}(t)>0\right\}, \quad i=0, \ldots, s, j=1, \ldots, q,
$$

where $a^{0}=\hat{a}$ (recall that $\hat{a}$ is given in Prop. 5.6), and

$$
\mathcal{M}_{j}=\bigcup_{i=0}^{s} \mathcal{M}_{j}^{i}, \quad j=1, \ldots, q .
$$

Let $i \in\{1, \ldots, s\}, j \in\{1, \ldots, q\}$. By Lemma 8.1, for the sequence $\left\{v_{j}\right\}$ and the set $\mathcal{M}_{j}^{i}$, there exist two sequences of sets $\left\{A_{j}^{i}\right\}$ and $\left\{B_{j}^{i}\right\}$ such that

$$
A_{j}^{i} \cup B_{j}^{i}=\mathcal{M}_{j}^{i}, \quad A_{j}^{i} \cap B_{j}^{i}=\emptyset, \quad \text { meas } B_{j}^{i} \rightarrow 0, \quad\left\|v_{j} \chi_{A_{j}^{i}}\right\|_{\infty}=o(\sqrt{\gamma})
$$

(so, in Lem. 8.1, we can set $\alpha:=O(\sqrt{\gamma})$ ). Let $\{\varepsilon\}$ be the sequence corresponding to $\{\delta w\}$ such that (6.15) holds. Set

$$
B=\left(\bigcup_{i=1}^{s} \bigcup_{j=1}^{q} B_{j}^{i}\right) \bigcup\left(\bigcup_{j=1}^{q}\left\{t \mid 0<\hat{a}_{j}(t)<\varepsilon\right\}\right) .
$$

Then in view of (9.3) and (9.4) we have: meas $B \rightarrow 0,\left\|v_{j} \chi_{\mathcal{M}_{j} \backslash B}\right\|_{\infty}=o(\sqrt{\gamma})$. Due to the hypothesis of linear independence of gradients $g_{j}^{\prime}(u)$, there exists a sequence of functions $\{\tilde{u}\}$ in $\mathcal{U}$ such that

$$
\begin{aligned}
& \|\tilde{u}\|_{\infty}=o(\sqrt{\gamma}), \tilde{u} \chi_{B}=0, \\
& g_{j}^{\prime}(u) \tilde{u} \chi_{\mathcal{M}_{j} \backslash B}=-v_{j} \chi_{\mathcal{M}_{j} \backslash B}, \\
& g_{j}^{\prime}(u) \tilde{u} \chi_{\left\{g_{j}(u)=0\right\} \backslash \mathcal{M}_{j}}=0, j=1, \ldots, q .
\end{aligned}
$$

Define a sequence $\left\{u^{\prime}\right\}$ by the relation $u^{\prime}=\delta u \chi_{B}$. Obviously, $\left\{u^{\prime}\right\} \in S^{\prime}$. Set $\delta u_{1}=\delta u-\tilde{u}-u^{\prime}, \delta w_{1}=\left(\delta u_{1}, \delta y\right)$. Then $\delta u_{1} \in \mathcal{S}_{u}$. Moreover, using (9.5), we deduce that, for the sequences $\{\delta w\},\left\{\delta w_{1}\right\}$, and $\left\{u^{\prime}\right\}$, the following relations hold

$$
\begin{aligned}
& \gamma(\delta w)=\gamma\left(\delta w_{1}\right)+\int_{0}^{T}\left|u^{\prime}\right|^{2} \mathrm{~d} t+o(\gamma(\delta w)), \\
& \Omega(\delta w, \lambda)=\Omega\left(\delta w_{1}, \lambda\right)+\int_{0}^{T}\left\langle\bar{H}_{u u}(w, p, a) u^{\prime}, u^{\prime}\right\rangle \mathrm{d} t+o(\gamma(\delta w)), \\
& \int_{0}^{T} a\left(g^{\prime}(u) \delta u\right)_{-} \mathrm{d} t \geq \int_{B} a\left(g^{\prime}(u) \delta u\right)_{-} \mathrm{d} t=\int_{0}^{T} a\left(g^{\prime}(u) u^{\prime}\right)_{-} \mathrm{d} t
\end{aligned}
$$

uniformly on $\Lambda_{0}$. Consequently,

$$
\begin{aligned}
\Phi_{C}^{1}(\delta w):= & \max _{\lambda \in M(C \Gamma)}\left\{\Omega(\delta w, \lambda)+\int_{0}^{T} a\left(g^{\prime}(u) \delta u\right)_{-} \mathrm{d} t\right\} \\
\geq & \max _{\lambda \in M(C \Gamma)}\left\{\Omega\left(\delta w_{1}, \lambda\right)\right. \\
& \left.+\int_{0}^{T}\left(\left\langle\bar{H}_{u u}(w, p, a) u^{\prime}, u^{\prime}\right\rangle+a\left(g^{\prime}(u) u^{\prime}\right)_{-}\right) \mathrm{d} t\right\}+o(\gamma(\delta w)) .
\end{aligned}
$$


Moreover, $\left\|\delta w_{1}\right\|_{\mathcal{W}} \rightarrow 0,\left\|\delta \dot{y}-f_{u}(w) \delta u_{1}-f_{y}(w) \delta y\right\|_{1}=o(\sqrt{\gamma(\delta w)})$, and relations (6.6)-(6.7) hold. Choose any $C^{\prime}<C$. Set $w^{\prime}=\left(0, u^{\prime}\right), \gamma\left(w^{\prime}\right)=\int_{0}^{T}\left|u^{\prime}\right|^{2} \mathrm{~d} t$. Then in view of Lemma 8.2 and definition (4.5) (of the functional $\left.\Omega_{M(C \Gamma)}\right)$ we get

$$
\begin{aligned}
& \max _{\lambda \in M(C \Gamma)}\left\{\Omega\left(\delta w_{1}, \lambda\right)+\int_{0}^{T}\left\langle\bar{H}_{u u}(w, p, a) u^{\prime}, u^{\prime}\right\rangle+\int_{0}^{T} a\left(g^{\prime}(u) \delta u^{\prime}\right)-\mathrm{d} t\right\} \\
& \geq \max _{\lambda \in M(C \Gamma)} \Omega\left(\delta w_{1}, \lambda\right)+C^{\prime} \gamma\left(w^{\prime}\right)=\Omega_{M(C \Gamma)}\left(\delta w_{1}\right)+C^{\prime} \gamma\left(w^{\prime}\right)
\end{aligned}
$$

starting from a certain number of the sequences. Combining this inequality with (9.9) we obtain

$$
\Phi_{C}^{1}(\delta w) \geq \Omega_{M(C \Gamma)}\left(\delta w_{1}\right)+C^{\prime} \gamma\left(w^{\prime}\right)+o(\gamma(\delta w)) .
$$

Furthermore, the relation $\left\|\delta \dot{y}-f^{\prime}(w) \delta w_{1}\right\|_{1}=o(\sqrt{\gamma(\delta w)})$ can be written as $\delta \dot{y}-f_{y}(w) \delta y-f_{u}(w) \delta u_{1}=\zeta$, $\|\zeta\|_{1}=o(\sqrt{\gamma(\delta w)})$. Let us define a sequence $\left\{y_{\zeta}\right\}$ by the equation $\dot{y}_{\zeta}=f_{y}(w) y_{\zeta}+\zeta, y_{\zeta}(0)=0$. Then $\left\|y_{\zeta}\right\|_{1,1}=$ $o(\sqrt{\gamma(\delta w)})$. Set $\delta y_{\mathcal{S}}=\delta y-y_{\zeta}, \delta w_{\mathcal{S}}=\left(\delta u_{1}, \delta y_{\mathcal{S}}\right)$. Then $\delta \dot{y}_{\mathcal{S}}-f^{\prime}(w) \delta w_{\mathcal{S}}=0$, and hence $\delta w_{\mathcal{S}} \in \mathcal{S}$ (see Def. (7.6) of the set $\mathcal{S})$. Since $\left\{\delta w_{1}\right\}$ satisfies relations (6.6)-(6.7), the same is true for $\left\{\delta w_{\mathcal{S}}\right\}$. Thus, we get

$$
\begin{aligned}
& \phi_{i}^{\prime}(\eta) \delta \eta_{\mathcal{S}} \leq o(\sqrt{\gamma(\delta w)}), i \in I_{\phi}(\eta) \cup\{0\}, \\
& \left|\phi_{i}^{\prime}(\eta) \delta \eta_{\mathcal{S}}\right|=o(\sqrt{\gamma(\delta w)}), i=r_{1}+1, \ldots, r, \\
& \delta w_{\mathcal{S}} \in \mathcal{S}, \quad\left\|\delta w_{\mathcal{S}}\right\|_{\mathcal{W}} \rightarrow 0
\end{aligned}
$$

where $\delta \eta_{\mathcal{S}}=\left(\delta y_{\mathcal{S}}(0), \delta y_{\mathcal{S}}(T)\right)$. By Lemma 7.2 , for each member $\delta w_{\mathcal{S}}$ of the sequence $\left\{\delta w_{\mathcal{S}}\right\}$ there exists a solution $\bar{w}=(\bar{u}, \bar{y})$ to the system

$$
\begin{aligned}
& \phi_{i}^{\prime}(\eta)\left(\delta \eta_{\mathcal{S}}+\bar{\eta}\right) \leq 0, i \in I_{\phi}(\eta) \cup\{0\}, \\
& \phi_{j}^{\prime}(\eta)\left(\delta \eta_{\mathcal{S}}+\bar{\eta}\right)=0, j=r_{1}+1, \ldots, r, \quad \bar{w} \in \mathcal{S}
\end{aligned}
$$

such that $\|\bar{w}\|_{\mathcal{W}}=o(\sqrt{\gamma(\delta w)})$, where $\bar{\eta}=(\bar{y}(0), \bar{y}(T))$. Set $\delta w_{\mathcal{K}}=\delta w_{\mathcal{S}}+\bar{w}$. Then for the sequence $\left\{\delta w_{\mathcal{K}}\right\}$ we obviously have

$$
\begin{aligned}
& \delta w_{\mathcal{K}} \in \mathcal{K}, \quad\left\|\delta w_{\mathcal{K}}\right\|_{\mathcal{W}} \rightarrow 0 \\
& \gamma\left(\delta w_{1}\right)=\gamma\left(\delta w_{\mathcal{K}}\right)+o(\gamma(\delta w)) \\
& \Omega_{M(C \Gamma)}\left(\delta w_{1}\right)=\Omega_{M(C \Gamma)}\left(\delta w_{\mathcal{K}}\right)+o(\gamma(\delta w)) .
\end{aligned}
$$

Now note that on the subspace $\mathcal{W}_{0}$ (specified by (7.1)) the functional $|\delta y(0)|^{2}+\int_{0}^{T}|\delta u(t)|^{2} \mathrm{~d} t$ is equivalent to the functional $\|\delta y\|_{\infty}^{2}+\int_{0}^{T}|\delta u(t)|^{2} \mathrm{~d} t$ (in the sense that the first functional estimates the second one on $\mathcal{W}_{0}$ from below and from above with certain positive multipliers) and by (3.1) the latter is equal to $\gamma(\delta w)$ in a small neighborhood of zero in $\mathcal{W}$. Since $\delta w_{\mathcal{K}} \in \mathcal{K}$, condition (4.6) of Theorem 4.1 implies that there exists $C_{\mathcal{K}}^{\prime}>0$ such that

$$
\Omega_{M(C \Gamma)}\left(\delta w_{\mathcal{K}}\right) \geq C_{\mathcal{K}}^{\prime} \gamma\left(\delta w_{\mathcal{K}}\right)
$$

starting from a certain member of the sequence $\left\{\delta w_{\mathcal{K}}\right\}$. Relations (9.10), (9.13), and (9.14) imply

$$
\Phi_{C}^{1}(\delta w) \geq C_{\mathcal{K}}^{\prime} \gamma\left(\delta w_{\mathcal{K}}\right)+C^{\prime} \gamma\left(w^{\prime}\right)+o(\gamma(\delta w)) .
$$

Recall that $\gamma\left(w^{\prime}\right)=\int_{0}^{T}\left|u^{\prime}\right|^{2} \mathrm{~d} t$, hence combining (9.6) with (9.12) we get

$$
\gamma(\delta w)=\gamma\left(\delta w_{\mathcal{K}}\right)+\gamma\left(w^{\prime}\right)+o(\gamma(\delta w)) .
$$


Consequently, the following relations hold

$$
\liminf \frac{\Phi_{C}^{1}(\delta w)}{\gamma(\delta w)} \geq \liminf \frac{C_{\mathcal{K}}^{\prime} \gamma\left(\delta w_{\mathcal{K}}\right)+C^{\prime} \gamma\left(w^{\prime}\right)}{\gamma\left(\delta w_{\mathcal{K}}\right)+\gamma\left(w^{\prime}\right)} \geq \min \left\{C_{\mathcal{K}}^{\prime}, C^{\prime}\right\}
$$

Since $\{\delta w\}$ is an arbitrary sequence of $S_{1}$, the above inequalities together with (6.18) implies $C_{\gamma}\left(\Phi_{C}^{1}, S_{1}\right) \geq$ $\min \left\{C_{\mathcal{K}}^{\prime}, C^{\prime}\right\}$. The latter holds for any $C^{\prime}<C$. Hence $C_{\gamma}\left(\Phi_{C}^{1}, S_{1}\right) \geq \min \left\{C_{\mathcal{K}}^{\prime}, C\right\}$. Combining this inequality with (6.12) and (6.17) we get $C_{\gamma}\left(\Psi_{\Lambda_{0}}, \Pi_{\sigma \gamma}\right) \geq \min \left\{C_{\mathcal{K}}^{\prime}, C\right\}>0$. In view of (5.11) and Proposition 3.7 this completes the proof of Theorem 4.1 .

\section{Proofs of Basic lemmas}

Here we give the proofs of Lemmas 6.2, 6.4, 7.2, 8.1, 8.2, and 8.3 used in the proof of Theorem 4.1.

\subsection{Proof of Lemma 6.2}

Consider the sequence $\{(\delta w, \varepsilon)\}$, where $\{\delta w\} \in \Pi_{o(\sqrt{\gamma})}$ (see (5.18)) and $\varepsilon \rightarrow+0$. For any member $(\delta w, \varepsilon)=$ $((\delta u, \delta y), \varepsilon)$ of the sequence $\{(\delta w, \varepsilon)\}$, we set

$$
\begin{aligned}
& \delta u_{\varepsilon}(t)= \begin{cases}\delta u(t) & \text { if } \quad|\delta u(t)|<\varepsilon, \\
0 & \text { otherwise, }\end{cases} \\
& \delta u^{\varepsilon}=\delta u-\delta u_{\varepsilon}, \quad \delta w_{\varepsilon}=\left(\delta u_{\varepsilon}, \delta y\right), \quad \delta w^{\varepsilon}=\left(\delta u^{\varepsilon}, 0\right) .
\end{aligned}
$$

Then $\left\|\delta u_{\varepsilon}\right\|_{\infty} \rightarrow 0$ and hence $\left\{\delta w_{\varepsilon}\right\} \in S^{\text {loc }}$. Moreover, $\{\delta w\}=\left\{\delta w_{\varepsilon}\right\}+\left\{\delta w^{\varepsilon}\right\}$. To continue the proof of the lemma, we need the following proposition.

Proposition 10.1. For the sequences $\{(\delta w, \varepsilon)\},\left\{\delta w_{\varepsilon}\right\}$, and $\left\{\delta w^{\varepsilon}\right\}$, the following formula holds

$$
\delta f=\delta_{\varepsilon} f+\delta^{\varepsilon} f+r_{f},
$$

where

$$
\begin{aligned}
& \delta f=f(w+\delta w)-f(w), \delta_{\varepsilon} f=f\left(w+\delta w_{\varepsilon}\right)-f(w), \\
& \delta^{\varepsilon} f=f\left(w+\delta w^{\varepsilon}\right)-f(w), r_{f}=\left(\bar{\delta}_{y} f-\delta_{y} f\right) \chi^{\varepsilon} \\
& \delta_{y} f=f(u, y+\delta y)-f(u, y) \\
& \bar{\delta}_{y} f=f(u+\delta u, y+\delta y)-f(u+\delta u, y)
\end{aligned}
$$

and $\chi^{\varepsilon}$ is a characteristic function of the set $\mathcal{M}^{\varepsilon}=\left\{t \mid \delta u^{\varepsilon}(t) \neq 0\right\}$.

Proof. We have

$$
\begin{aligned}
\delta f & =\delta f \chi^{\varepsilon}+\delta f\left(1-\chi^{\varepsilon}\right) \\
& =\left(f\left(u+\delta u^{\varepsilon}, y+\delta y\right)-f\left(u+\delta u^{\varepsilon}, y\right)+\delta^{\varepsilon} f\right) \chi^{\varepsilon}+\delta_{\varepsilon} f\left(1-\chi^{\varepsilon}\right) \\
& =\bar{\delta}_{y} f \chi^{\varepsilon}+\delta^{\varepsilon} f+\delta_{\varepsilon} f-\delta_{\varepsilon} f \chi^{\varepsilon}=\delta^{\varepsilon} f+\delta_{\varepsilon} f+\left(\bar{\delta}_{y} f-\delta_{y} f\right) \chi^{\varepsilon} \\
& =\delta_{\varepsilon} f+\delta^{\varepsilon} f+r_{f} .
\end{aligned}
$$

Let us continue the proof of the lemma. Note that from the definition of $r_{f}$ in (10.2) it follows that

$$
\left\|r_{f}\right\|_{\infty} \leq\left\|\delta_{y} f\right\|_{\infty}+\left\|\bar{\delta}_{y} f\right\|_{\infty} \leq \text { const. }\|\delta y\|_{\infty} \rightarrow 0,
$$


where const. $>0$ does not depend on the member of the sequence $\{(\delta w, \varepsilon)\}$. Obviously, for a given sequence $\{\delta w\} \in \Pi_{o(\sqrt{\gamma})}$, one can choose a sequence $\varepsilon \rightarrow+0$ such that

$$
(a) \frac{\left\|r_{f}\right\|_{\infty}}{\varepsilon^{2}} \rightarrow 0, \quad(b) \frac{\sqrt{\gamma(\delta w)}}{\varepsilon^{2}} \rightarrow 0 .
$$

(Note that $(b) \Rightarrow(a)$, since $\|\delta y\|_{\infty} \leq \sqrt{\gamma(\delta w)}$.) Then we have

$$
\left\|r_{f}\right\|_{1} \leq \frac{\left\|r_{f}\right\|_{\infty}}{\varepsilon^{2}} \int_{\mathcal{M}^{\varepsilon}} \varepsilon^{2} \mathrm{~d} t \leq \frac{\left\|r_{f}\right\|_{\infty}}{\varepsilon^{2}} \int_{\mathcal{M}^{\varepsilon}}|\delta u|^{2} \mathrm{~d} t=o\left(\gamma^{\varepsilon}\right),
$$

where $\gamma^{\varepsilon}:=\gamma\left(\delta w^{\varepsilon}\right)$. Let $\lambda=(\mu, p, a) \in \Lambda_{0}$. Since $\delta H=p \delta f$, it follows from Proposition 10.1 and estimate (10.3) that $\int_{0}^{T} \delta H \mathrm{~d} t=\int_{0}^{T} \delta_{\varepsilon} H \mathrm{~d} t+\int_{0}^{T} \delta^{\varepsilon} H \mathrm{~d} t+o\left(\gamma^{\varepsilon}\right)$ uniformly on $\Lambda_{0}$, where $\delta_{\varepsilon} H=H\left(u+\delta u_{\varepsilon}, y+\delta y, p\right)-H(u, y, p)$, $\delta^{\varepsilon} H=H\left(u+\delta u^{\varepsilon}, y, p\right)-H(u, y, p)$. Hence, from definition (5.1) of the function $\Psi$, we obtain: $\Psi(\delta w, \lambda)=$ $\Psi\left(\delta w_{\varepsilon}, \lambda\right)+\int_{0}^{T} \delta^{\varepsilon} H \mathrm{~d} t+o\left(\gamma^{\varepsilon}\right)$ uniformly on $\Lambda_{0}$, and then

$$
\Psi_{\Lambda_{0}}(\delta w)=\max _{\lambda \in \Lambda_{0}}\left\{\Psi\left(\delta w_{\varepsilon}, \lambda\right)+\int_{0}^{T} \delta^{\varepsilon} H \mathrm{~d} t\right\}+o\left(\gamma^{\varepsilon}\right) .
$$

Furthermore, the condition $g(u+\delta u) \leq 0$ implies

$$
g\left(u+\delta u_{\varepsilon}\right) \leq 0, \quad g\left(u+\delta u^{\varepsilon}\right) \leq 0 .
$$

Consequently, by (3.6), $\delta^{\varepsilon} H \geq C \Gamma\left(\delta u^{\varepsilon}\right)$ for any $\lambda \in M(C \Gamma)$, and then $\int_{0}^{T} \delta^{\varepsilon} H \mathrm{~d} t \geq C \gamma^{\varepsilon}$ for any $\lambda \in M(C \Gamma)$. Since $M(C \Gamma) \subset \Lambda_{0}$, we have

$$
\begin{aligned}
\max _{\lambda \in \Lambda_{0}}\left\{\Psi\left(\delta w_{\varepsilon}, \lambda\right)+\int_{0}^{T} \delta^{\varepsilon} H \mathrm{~d} t\right\} & \geq \max _{\lambda \in M(C \Gamma)}\left\{\Psi\left(\delta w_{\varepsilon}, \lambda\right)+\int_{0}^{T} \delta^{\varepsilon} H \mathrm{~d} t\right\} \\
& \geq \max _{\lambda \in M(C \Gamma)}\left\{\Psi\left(\delta w_{\varepsilon}, \lambda\right)+C \gamma^{\varepsilon}\right\}=\Psi_{M(C \Gamma)}\left(\delta w_{\varepsilon}\right)+C \gamma^{\varepsilon}
\end{aligned}
$$

(see Def. (6.10) of $\left.\Psi_{M(C \Gamma)}\right)$. This along with (10.4) implies

$$
\Psi_{\Lambda_{0}}(\delta w) \geq \Psi_{M(C \Gamma)}\left(\delta w_{\varepsilon}\right)+C \gamma^{\varepsilon}+o\left(\gamma^{\varepsilon}\right) .
$$

Moreover, we obviously have $\gamma=\gamma_{\varepsilon}+\gamma^{\varepsilon}$, where $\gamma:=\gamma(\delta w), \gamma_{\varepsilon}:=\gamma\left(\delta w_{\varepsilon}\right), \gamma^{\varepsilon}:=\gamma\left(\delta w^{\varepsilon}\right)$. For the sequence of pairs $\{(\delta w, \varepsilon)\}$, let us consider two cases.

Case 1. $\liminf \gamma_{\varepsilon} / \gamma=0$. In this case, we choose a subsequence such that $\gamma_{\varepsilon}=o(\gamma)$, and hence $\gamma^{\varepsilon} / \gamma \rightarrow 1$ for this subsequence. Without loss of generality assume that this condition is satisfied for the whole sequence $\{\delta w\}$. Then using Lemma 6.1 and the inequality $a \delta_{\varepsilon} g_{-} \geq 0$ we get

$$
\begin{aligned}
\Psi_{M(C \Gamma)}\left(\delta w_{\varepsilon}\right) & =\max _{\lambda \in M(C)}\left\{\Omega\left(\delta w_{\varepsilon}, \lambda\right)+\int_{0}^{T} a \delta_{\varepsilon} g_{-} \mathrm{d} t\right\}+o\left(\gamma_{\varepsilon}\right) \\
& \geq \Omega_{M(C \Gamma)}\left(\delta w_{\varepsilon}\right)+o\left(\gamma_{\varepsilon}\right)
\end{aligned}
$$

(see Def. (4.5) of $\Omega_{M(C \Gamma)}$ ). Combining (10.6) and (10.7) we obtain

$$
\Psi_{\Lambda_{0}}(\delta w) \geq \Omega_{M(C \Gamma)}\left(\delta w_{\varepsilon}\right)+C \gamma^{\varepsilon}+o(\gamma) .
$$


Since $\left|\Omega_{M(C \Gamma)}\left(\delta w_{\varepsilon}\right)\right| \leq O\left(\gamma_{\varepsilon}\right)=o(\gamma)$ and $\gamma^{\varepsilon} / \gamma \rightarrow 1$, we get

$$
\liminf \frac{\Psi_{\Lambda_{0}}(\delta w)}{\gamma(\delta w)} \geq C
$$

Case 2. $\liminf \gamma_{\varepsilon} / \gamma>0$, and hence $\gamma \leq O\left(\gamma_{\varepsilon}\right)$ and $\gamma^{\varepsilon} \leq O\left(\gamma_{\varepsilon}\right)$. Let us show, in this case, that

$$
\left\{\delta w_{\varepsilon}\right\} \in S_{o(\sqrt{\gamma})}^{\operatorname{loc}}
$$

Indeed, the sequence $\left\{\delta w_{\varepsilon}\right\}=\left\{\left(\delta u_{\varepsilon}, \delta y\right)\right\}$ satisfies the conditions $\left\|\delta u_{\varepsilon}\right\|_{\infty}+\|\delta y\|_{1,1} \rightarrow 0$, i.e., $\left\{\delta w_{\varepsilon}\right\} \in S^{\text {loc }}$, and moreover, $\varphi\left(u+\delta u_{\varepsilon}\right) \leq 0$. Furthermore, since $\sqrt{\gamma} / \varepsilon^{2} \rightarrow 0$, the following estimate holds

$$
\text { meas } \mathcal{M}^{\varepsilon}=\frac{1}{\varepsilon^{2}} \int_{\mathcal{M}^{\varepsilon}} \varepsilon^{2} \mathrm{~d} t \leq \frac{1}{\varepsilon^{2}} \gamma^{\varepsilon} \cdot O(1) \leq \frac{\sqrt{\gamma}}{\varepsilon^{2}} \sqrt{\gamma^{\varepsilon}} \cdot O(1)=o\left(\sqrt{\gamma^{\varepsilon}}\right)
$$

In virtue of Proposition $10.1 \delta f=\delta_{\varepsilon} f+\delta^{\varepsilon} f+r_{f}$, where $\left\|r_{f}\right\|_{1}=o\left(\gamma^{\varepsilon}\right)$ (see (10.3)) and $\left\|\delta^{\varepsilon} f\right\|_{1}=O\left(\right.$ meas $\left.\mathcal{M}^{\varepsilon}\right)=$ $o\left(\sqrt{\gamma^{\varepsilon}}\right)$ (see $\left.(10.10)\right)$. This and the relations $\|\delta \dot{y}-\delta f\|_{1}=o(\sqrt{\gamma}), \gamma=O\left(\gamma_{\varepsilon}\right)$ imply $\left\|\delta \dot{y}-\delta_{\varepsilon} f\right\|_{1}=o\left(\sqrt{\gamma_{\varepsilon}}\right)$. Note that in the definition of $\delta w_{\varepsilon}=\left(\delta u_{\varepsilon}, \delta y\right)$ the component $\delta y$ is the same as in $\delta w$. Therefore, conditions $\sigma(\delta w)=o(\sqrt{\gamma})$ and $\gamma \leq O\left(\gamma_{\varepsilon}\right)$ imply $\sigma\left(\delta w_{\varepsilon}\right)=o\left(\sqrt{\gamma_{\varepsilon}}\right)$. Finally, $\int_{0}^{T} \hat{a} \delta_{\varepsilon} g_{-} \mathrm{d} t \leq \int_{0}^{T} \hat{a} \delta g_{-} \mathrm{d} t \leq O(\gamma)=O\left(\gamma_{\varepsilon}\right)$. Thus, (10.9) is proved.

It follows from (10.9) and inequality (10.6) that

$$
\begin{aligned}
\liminf \frac{\Psi_{\Lambda_{0}}(\delta w)}{\gamma(\delta w)} & \geq \liminf \frac{\Psi_{M(C \Gamma)}\left(\delta w_{\varepsilon}\right)+C \gamma^{\varepsilon}}{\gamma} \\
& =\liminf \left(\frac{\gamma_{\varepsilon}}{\gamma} \cdot \frac{\Psi_{M(C \Gamma)}\left(\delta w_{\varepsilon}\right)}{\gamma_{\varepsilon}}+C \cdot \frac{\gamma^{\varepsilon}}{\gamma}\right) \geq \liminf \left(\min \left\{\frac{\Psi_{M(C \Gamma)}\left(\delta w_{\varepsilon}\right)}{\gamma_{\varepsilon}}, C\right\}\right) \\
& =\min \left\{\liminf \frac{\Psi_{M(C \Gamma)}\left(\delta w_{\varepsilon}\right)}{\gamma_{\varepsilon}}, C\right\} \geq \min \left\{\inf _{S_{o(\sqrt{\gamma})}^{\operatorname{loc}}}\left(\liminf \frac{\Psi_{M(C \Gamma)}}{\gamma}\right), C\right\} \\
& =\min \left\{C_{\gamma}\left(\Psi_{M(C \Gamma)}, S_{o(\sqrt{\gamma})}^{\operatorname{loc}}\right), C\right\},
\end{aligned}
$$

where $\gamma=\gamma(\delta w)$.

Thus, we have proved that for any sequence $\{\delta w\} \in \Pi_{o(\sqrt{\gamma})}$ there exists a subsequence such that for the subsequence we have:

$$
\liminf \Psi_{\Lambda_{0}} / \gamma \geq \min \left\{C_{\gamma}\left(\Psi_{M(C \Gamma)}, S_{o(\sqrt{\gamma})}^{\mathrm{loc}}\right), C\right\}
$$

This implies inequality (6.11). Indeed, in the opposite case there exists a sequence $\{\delta w\} \in \Pi_{o(\sqrt{\gamma})}$ such that

$$
\liminf \Psi_{\Lambda_{0}} / \gamma<\min \left\{C_{\gamma}\left(\Psi_{M(C \Gamma)}, S_{o(\sqrt{\gamma})}^{\operatorname{loc}}\right), C\right\}
$$

Consequently, there exists a subsequence such that

$$
\lim \Psi_{\Lambda_{0}} / \gamma<\min \left\{C_{\gamma}\left(\Psi_{M(C \Gamma)}, S_{o(\sqrt{\gamma})}^{\mathrm{loc}}\right), C\right\}
$$

But then the choice of a subsequence satisfying (10.11) is impossible. The lemma is proved. 


\subsection{Proof of Lemma 6.4}

Take any sequence $\{\delta w\} \in S_{o(\sqrt{\gamma})}^{\text {loc }}$. As it was noted in Section 6.1, this sequence satisfies relations (6.3) and (6.5)-(6.8). From the relation $g(u+\delta u) \leq 0$ (see (6.3)) it follows that

$$
g_{j}(u(t))+g_{j}^{\prime}(u(t)) \delta u(t) \leq k_{1}|\delta u(t)|^{2}, j=1, \ldots, q,
$$

where $k_{1}>0$ does not depend on the member of the sequence. Due to the hypothesis of linear independence of the gradients $g_{j}^{\prime}(u)$, for any sequence $\varepsilon=\varepsilon(\delta u) \rightarrow+0$, there exist $k_{2}>0$ and a sequence of corrections $\{\bar{u}\}$ such that

$$
\begin{aligned}
& |\bar{u}(t)| \leq k_{2}\left(|\delta u(t)|^{2}+\sum_{j=1}^{q} \chi_{\left\{\hat{a}_{j} \geq \varepsilon\right\}}(t)\left(g_{j}^{\prime}(u(t)) \delta u(t)\right)_{-}\right), \\
& g_{j}(u(t))+g_{j}^{\prime}(u(t))(\delta u(t)+\bar{u}(t)) \leq 0 \text { a.e., } j=1, \ldots, q, \\
& g_{j}^{\prime}(u(t))(\delta u(t)+\bar{u}(t))=0 \text { if } \quad \hat{a}_{j}(t) \geq \varepsilon, j=1, \ldots, q, \\
& g_{j}^{\prime}(u(t)) \bar{u}(t)=0 \text { if } 0<\hat{a}_{j}(t)<\varepsilon, j=1, \ldots, q .
\end{aligned}
$$

Relations (10.13) imply $\|\bar{u}\|_{\infty} \leq O\left(\|\delta u\|_{\infty}\right)=o(1)$. Since

$$
\int_{\left\{\hat{a}_{j} \geq \varepsilon\right\}}\left(g_{j}^{\prime}(u) \delta u\right)_{-} \mathrm{d} t \leq \frac{1}{\varepsilon} \int_{0}^{T} \hat{a}_{j}\left(g_{j}^{\prime}(u) \delta u\right)_{-} \mathrm{d} t \leq \frac{1}{\varepsilon} O(\gamma(\delta w))
$$

(see (6.8)), relation (10.13) implies that $\|\bar{u}\|_{1} \leq O(\gamma(\delta w)) / \varepsilon$. Choose $\varepsilon=\varepsilon(\delta w) \rightarrow+0$ such that $\|\delta w\|_{\infty} / \varepsilon \rightarrow 0$. Then $\sqrt{\gamma(\delta w)} / \varepsilon \rightarrow 0$, and hence $\|\bar{u}\|_{1}=o(\sqrt{\gamma(\delta w)})$. Moreover, since $\|\delta u\|_{\infty} / \varepsilon \rightarrow 0$, we have

$$
\begin{aligned}
& \int_{0}^{T}|\bar{u}|^{2} \mathrm{~d} t \leq\|\bar{u}\|_{\infty}\|\bar{u}\|_{1} \leq O\left(\|\delta u\|_{\infty}\right) \cdot O(\gamma(\delta w)) / \varepsilon=o(\gamma(\delta w)), \\
& \int_{0}^{T}|\delta u| \cdot|\bar{u}| \mathrm{d} t \leq\|\delta u\|_{\infty}\|\bar{u}\|_{1} \leq\|\delta u\|_{\infty} O(\gamma(\delta w)) / \varepsilon=o(\gamma(\delta w)), \\
& \int_{0}^{T}|\delta y| \cdot|\bar{u}| \mathrm{d} t \leq\|\delta y\|_{\infty}\|\bar{u}\|_{1} \leq\|\delta y\|_{\infty} O(\gamma(\delta w)) / \varepsilon=o(\gamma(\delta w)) .
\end{aligned}
$$

Set $\left\{\delta w_{1}\right\}=\{(\delta u+\bar{u}, \delta y)\}$. Relations (10.17)-(10.19) imply that, for the sequences $\left\{\delta w_{1}\right\}$ and $\{\delta w\}$, we have

$$
\Omega\left(\delta w_{1}, \lambda\right)=\Omega(\delta w, \lambda)+o(\gamma(\delta w)), \quad \gamma\left(\delta w_{1}\right)=\gamma(\delta w)+o(\gamma(\delta w))
$$

uniformly on $\Lambda_{0}$. Since $\|\bar{u}\|_{\infty} \rightarrow 0$, we have $\left\|\delta w_{1}\right\|_{\mathcal{W}} \rightarrow 0$, and using $(6.5)$ and the estimate $\|\bar{u}\|_{1}=o(\sqrt{\gamma(\delta w)})$ we get

$$
\left\|\delta \dot{y}-f^{\prime}(w) \delta w_{1}\right\|_{1}=o(\sqrt{\gamma}) .
$$

Moreover, the sequence $\left\{\delta w_{1}\right\}$ satisfies relations (6.6)-(6.7) (with $\delta \eta=\delta \eta_{1}$ since $\delta y=\delta y_{1}$ ) and the relations

$$
\begin{aligned}
& g_{j}(u(t))+g_{j}^{\prime}(u(t)) \delta u_{1}(t) \leq 0 \text { a.e., } j=1, \ldots, q, \\
& g_{j}^{\prime}(u(t)) \delta u_{1}(t)=0 \text { if } \hat{a}_{j}(t) \geq \varepsilon, j=1, \ldots, q, \\
& g_{j}^{\prime}(u(t)) \delta u(t)=g_{j}^{\prime}(u(t)) \delta u_{1}(t) \text { if } 0<\hat{a}_{j}(t)<\varepsilon, j=1, \ldots, q .
\end{aligned}
$$


Consequently $\left\{\delta w_{1}\right\} \in S_{1}$. Let us show that, for any $j=1, \ldots, q$, we have

$$
\int_{0}^{T} a_{j}\left(g_{j}^{\prime}(u) \delta u_{1}\right)_{-} \mathrm{d} t \leq \int_{0}^{T} a_{j}\left(g_{j}(u+\delta u)\right)_{-} \mathrm{d} t+o(\gamma)
$$

uniformly on $\Lambda_{0}$. Indeed, since $\varepsilon \rightarrow 0$ and in view of (5.16) $\left\{t \mid \hat{a}_{j}(t) \leq \varepsilon\right\} \subset\left\{t \mid a_{j}(t) \leq \hat{C} \varepsilon\right\}$, relations (10.23) and (10.24) imply

$$
\begin{aligned}
\int_{0}^{T} a_{j}\left(g_{j}^{\prime}(u) \delta u_{1}\right)_{-} \mathrm{d} t & =\int_{\hat{a}_{j} \leq \varepsilon} a_{j}\left(g_{j}^{\prime}(u) \delta u_{1}\right)_{-} \mathrm{d} t \\
& =\int_{\hat{a}_{j} \leq \varepsilon} a_{j}\left(g_{j}^{\prime}(u) \delta u\right)_{-} \mathrm{d} t \leq \int_{a_{j} \leq \hat{C} \varepsilon} a_{j}\left(g_{j}^{\prime}(u) \delta u\right)_{-} \mathrm{d} t \\
& \leq \int_{a_{j} \leq \hat{C} \varepsilon} a_{j}\left(g_{j}(u+\delta u)\right)_{-} \mathrm{d} t+\varepsilon O(\gamma(\delta w)) \\
& \leq \int_{0}^{T} a_{j}\left(g_{j}(u+\delta u)\right)_{-} \mathrm{d} t+o(\gamma(\delta w))
\end{aligned}
$$

From relations (6.9), (6.10), (6.16), (10.20), and (10.25) it follows that $\Phi_{C}^{1}\left(\delta w_{1}\right) \leq \Psi_{M(C \Gamma)}(\delta w)+o(\gamma(\delta w))$, where $\left\{\delta w_{1}\right\} \in S_{1}$. Along with the second relation in (10.20) this implies that

$$
\liminf \frac{\Psi_{M(C \Gamma)}(\delta w)}{\gamma(\delta w)} \geq \liminf \frac{\Phi_{C}^{1}\left(\delta w_{1}\right)}{\gamma\left(\delta w_{1}\right)} \geq C_{\gamma}\left(\Phi_{C}^{1}, S_{1}\right)
$$

Since $\{\delta w\}$ is an arbitrary sequence in $S_{o(\sqrt{\gamma})}^{\text {loc }}$, inequality (6.17) follows. The lemma is proved.

\subsection{Proof of Lemma 7.2}

In order to prove Lemma 7.2 we need five auxiliary propositions, which will be proved bellow. Recall that the critical cone $\mathcal{K}$ was defined by relations (4.1)-(4.3) and the support $\mathcal{S}$ was defined by (7.6).

Proposition 10.2. The following equality holds

$$
\mathcal{K}=\{\delta w \in \mathcal{S} \mid \text { conditions }(4.1) \text { hold }\}
$$

Proof. Let $\mathcal{K}_{\mathcal{S}}$ denote the r.h.s. of equality (10.26). Since $\mathcal{K} \subset \mathcal{R}_{s}=\mathcal{S}$ and each element of $\mathcal{K}$ satisfies conditions (4.1), we have $\mathcal{K} \subset \mathcal{K}_{\mathcal{S}}$. Vice versa, each element of $\mathcal{K}_{\mathcal{S}}$ satisfies all equalities and inequalities in $(7.7),(4.1)$, and $(4.2)$, and hence belongs to $\mathcal{K}$. It means that $\mathcal{K}_{\mathcal{S}} \subset \mathcal{K}$. Consequently, $\mathcal{K}=\mathcal{K}_{\mathcal{S}}$.

Recall that the cone $Q$ (defined in (7.4)) and the system of functionals $w_{1}^{*}, \ldots, w_{s}^{*}$ were introduced in Section 7 . Let us note that from the maximality of this system we get the following important property of the cone $\mathcal{S}$.

Proposition 10.3. If a linear functional $w^{*} \in Q$ has an integral representation $\left\langle w^{*}, \delta w\right\rangle=-\int_{0}^{T} a g^{\prime}(u) \delta u \mathrm{~d} t$ on the cone $\mathcal{S}$ such that $a \in L^{1}\left(0, T ; \mathbb{R}^{q *}\right), a \geq 0$, and ag $(u)=0$, then $\left\langle w^{*}, \delta w\right\rangle=0$ for all $\delta w \in \mathcal{S}$.

Recall that $\mathcal{S}_{u}$ was defined by (7.7), and the sets $\mathcal{M}_{j}$ were defined by (9.2). Obviously, $\mathcal{M}_{j}=\{t \mid$ $\left.\sum_{i=0}^{s} a_{j}^{i}(t)>0\right\}, j=1, \ldots, q$. Set

$$
\mathcal{N}_{j}=\left\{t \mid g_{j}(u(t))=0\right\} \backslash \mathcal{M}_{j}, \quad j=1, \ldots, q .
$$


Proposition 10.4. The following equality holds

$$
\mathcal{S}_{u}=\left\{\delta u \in \mathcal{U} \mid g_{j}^{\prime}(u) \delta u \chi_{\mathcal{N}_{j}} \leq 0, g_{j}^{\prime}(u) \delta u \chi_{\mathcal{M}_{j}}=0, j=1, \ldots, q\right\}
$$

where $\chi_{\mathcal{N}_{j}}$ and $\chi_{\mathcal{M}_{j}}$ are the characteristic functions of the sets $\mathcal{N}_{j}$ and $\mathcal{M}_{j}$, respectively, $j=1, \ldots, q$.

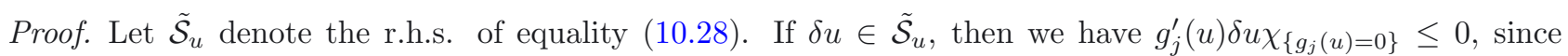
$\mathcal{M}_{j} \cup \mathcal{N}_{j}=\left\{t \mid g_{j}(u(t))=0\right\} \forall j$. Moreover, for any $j$, the condition $g_{j}^{\prime}(u) \delta u \chi_{\mathcal{M}_{j}}=0$ implies $a_{j}^{i} g_{j}^{\prime}(u) \delta u=0$ $\forall i$ and hence $a^{i} g^{\prime}(u) \delta u=0 \forall i$. Consequently, $\delta u \in \mathcal{S}_{u}$. Vice versa, let $\delta u \in \mathcal{S}_{u}$. For any $i$, the condition

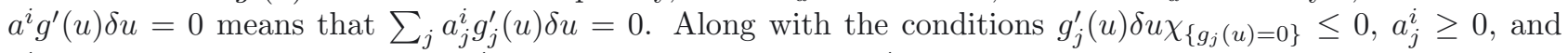
$a_{j}^{i} g_{j}(u)=0 \forall j$ this implies that $a_{j}^{i} g_{j}^{\prime}(u) \delta u=0$ and hence $\sum_{i} a_{j}^{i} g_{j}^{\prime}(u) \delta u=0 \forall j$. Consequently, $g_{j}^{\prime}(u) \delta u \chi_{\mathcal{M}_{j}}=0$ $\forall j$. Then from the conditions $g_{j}^{\prime}(u) \delta u \chi_{\left\{g_{j}(u)=0\right\}} \leq 0 \forall j$ it follows that $g_{j}^{\prime}(u) \delta u \chi_{\mathcal{N}_{j}} \leq 0 \forall j$. Consequently, $\delta u \in \tilde{\mathcal{S}}_{u}$. Thus, $\mathcal{S}_{u}=\tilde{\mathcal{S}}_{u}$.

In the space $L^{1}\left(0, T ; \mathbb{R}^{m *}\right)$, consider the cone $\mathcal{C}$ of functions $h$ of the form $h=-a g^{\prime}(u)$, where $a \in L^{1}\left(0, T ; \mathbb{R}^{q *}\right)$ is such that

$$
a_{j}(t)=0 \text { if } t \notin \mathcal{M}_{j} \cup \mathcal{N}_{j} ; \quad a_{j}(t) \geq 0 \text { if } t \in \mathcal{N}_{j}, j=1, \ldots, q .
$$

Proposition 10.5. $\mathcal{C}^{*}=\mathcal{S}_{u}$, where $\mathcal{C}^{*}$ is the cone polar to $C$.

Proof. (a) Let us show that $\mathcal{S}_{u} \subset \mathcal{C}^{*}$. Indeed, if $\delta u \in \mathcal{S}_{u}$ and $h \in \mathcal{C}$, then from (10.28) and (10.29) we get $\langle\delta u, h\rangle=\int_{0}^{T} h \delta u \mathrm{~d} t=-\int_{0}^{T} \sum_{j=1}^{q} a_{j} g_{j}^{\prime}(u) \delta u \mathrm{~d} t \geq 0$. (b) Let us show that $\mathcal{C}^{*} \subset \mathcal{S}_{u}$. Let $\delta u \in \mathcal{C}^{*}, i . e$., $\delta u \in L^{\infty}\left(0, T ; \mathbb{R}^{m}\right)$ and for any $h \in \mathcal{C}$ we have $\int_{0}^{T} h \delta u \mathrm{~d} t=-\sum_{j=1}^{q} \int_{0}^{T} a_{j} g_{j}^{\prime}(u) \delta u \mathrm{~d} t \geq 0$, where $a \in L^{1}\left(0, T ; \mathbb{R}^{q *}\right)$ satisfies (10.29). This implies that $g_{j}^{\prime}(u) \delta u \chi_{\mathcal{N}_{j}} \leq 0$ and $g_{j}^{\prime}(u) \delta u \chi_{\mathcal{M}_{j}}=0, j=1, \ldots, q, i . e ., \delta u \in \mathcal{S}_{u}$.

Now set

$$
\Sigma=\left\{\left(\delta u, \delta y_{0}\right) \in \mathcal{U} \times \mathbb{R}^{n} \mid \delta u \in \mathcal{S}_{u}\right\}
$$

Obviously, there is a one-to-one correspondence between the sets $\mathcal{S}$ (defined in (7.6)) and $\Sigma$ given by the mapping $(\delta u, \delta y) \in \mathcal{S} \rightarrow(\delta u, \delta y(0)) \in \Sigma$.

Let $\mu=\left(\mu_{0}, \ldots, \mu_{r}\right) \in \mathbb{R}^{(r+1) *}, \varphi^{\mu}=\sum_{i=0}^{r} \mu_{i} \phi_{i}$, and let $\varphi_{\eta}^{\mu}(\eta) \delta \eta$ be the functional (defined on the subspace $\left.\mathcal{W}_{0}\right)$ introduced in Section 7 . In what follows, it will be convenient to treat $\varphi_{\eta}^{\mu}(\eta) \delta \eta$ as a functional defined on $\Sigma$. Namely, define a linear functional

$$
\left(\delta u, \delta y_{0}\right) \in \mathcal{U} \times \mathbb{R}^{n} \rightarrow \varphi_{y_{0}}^{\mu}(\eta) \delta y_{0}+\varphi_{y_{T}}^{\mu}(\eta) \delta y(T)
$$

where $\delta y$ is the solution to the equation $\delta \dot{y}=f_{u}(w) \delta u+f_{y}(w) \delta y$ with the initial condition $\delta y(0)=\delta y_{0}$. For this functional, we shall use the same notation $\varphi_{\eta}^{\mu}(\eta) \delta \eta$. This will also concern elements of $Q$. Set

$$
P=\left\{(h, \xi) \in L^{1}\left(0, T ; \mathbb{R}^{m *}\right) \times \mathbb{R}^{n *} \mid h \in \mathcal{C}, \xi=0\right\}
$$

Since by Proposition $10.5, \mathcal{C}^{*}=\mathcal{S}_{u}$, we obviously have

$$
P^{*}=\Sigma
$$

Moreover, each element of $P$ can be treated as a functional on $\mathcal{U} \times \mathbb{R}^{n}$ with a pure integral representation. The following property will play a crucial role for the existence of corresponding Hoffman's error bounds.

Proposition 10.6. There exists a subspace $L$ in $P$ such that $P \cap Q \subset L \subset P$.

Proof. Let $l \in P \cap Q$. Then $l$ has an integral representation: $l\left(\delta u, \delta y_{0}\right)=-\int_{0}^{T} a g^{\prime}(u) \delta u \mathrm{~d} t$, where $a \in$ $L^{1}\left(0, T ; \mathbb{R}^{q *}\right)$ satisfies $(10.29)$. Set $\tilde{a}_{j}=a_{j} \chi_{\mathcal{N}_{j}} \forall j, \tilde{a}=\left(\tilde{a}_{1}, \ldots, \tilde{a}_{q}\right)$. Then, obviously, we have $l(\delta w)=$ $-\int_{0}^{T} \tilde{a} g^{\prime}(u) \delta u \mathrm{~d} t$ on $\mathcal{S}$. Moreover, $\tilde{a} \geq 0, \tilde{a} g(u)=0$, and $\tilde{a}_{j} \chi_{\mathcal{N}_{j}}=\tilde{a}_{j} \forall j$. Since $l \in Q$, by Proposition $10.3, l$ is 
equal to zero on $\mathcal{S}$ and hence $\int_{0}^{T} \tilde{a} g^{\prime}(u) \delta u \mathrm{~d} t=0$ on $\mathcal{S}_{u}$. This implies that $\tilde{a}=0$. Consequently, $a_{j} \chi \mathcal{N}_{j}=0 \forall j$. The latter conditions define a subspace $L$ in $P$ (see (10.29) and (10.32)).

Now we can prove Lemma 7.2. The proof will be based on abstract Lemma 11.9 proved in Section 11.

Proof of Lemma 7.2. For any $\delta w \in \mathcal{S}$ consider system (7.8) of linear inequalities and equalities on the cone $\Sigma$ (i.e., with $\left(\bar{u}, \bar{y}_{0}\right) \in \Sigma$ ). According to Proposition 10.6 and since $P^{*}=\Sigma$, this system on $\Sigma$ satisfies all conditions of Lemma 11.9 (with $X=\mathcal{U} \times \mathbb{R}^{n}, Y=L^{1}\left(0, T ; \mathbb{R}^{m *}\right) \times \mathbb{R}^{n *}, K=\Sigma, K_{Y}=P, K_{l m}=Q$ ). By this lemma, for any $\delta w \in \mathcal{S}$, system (7.8) is compatible and has Hoffman's error bound. Consequently, the same system considered on the cone $\mathcal{S}$ (i.e., with $\bar{w}=(\bar{u}, \bar{y}) \in \mathcal{S})$ is always compatible and has Hoffman's error bound too (note that a finite dimensional subspace in Def. 11.8 can be equipped with the norm of the space $\mathcal{W}$ ). The lemma is proved.

\subsection{Proof of Lemma 8.1}

Recall that at the beginning of Section $8, L_{+}^{p}(0, T ; \mathbb{R})$ was defined as the cone of nonnegative functions in $L^{p}(0, T ; \mathbb{R})$. The following proposition will be used in the proof of Lemma 8.1.

Proposition 10.7. Let $r \in L_{+}^{\infty}(0, T ; \mathbb{R}), \mathcal{M}=\{t \mid r(t)>0\}, v \in L_{+}^{1}(0, T ; \mathbb{R})$, and $\mathcal{N}=\{t \mid v(t)>0\}$. Assume that $\mathcal{N} \subset \mathcal{M}$. For $\delta>0$, set

$$
r_{\delta}(t)=\left\{\begin{array}{ll}
r(t) & \text { if } \quad r(t)<\delta \\
0 & \text { otherwise },
\end{array} \quad r^{\delta}(t)=r(t)-r_{\delta}(t),\right.
$$

and similarly, for $\varepsilon>0$, define $v_{\varepsilon}(t)$ and $v^{\varepsilon}(t)$. Set $\mathcal{M}_{\delta}=\left\{t \mid r_{\delta}(t)>0\right\}, \mathcal{M}^{\delta}=\left\{t \mid r^{\delta}(t)>0\right\}, \mathcal{N}_{\varepsilon}=\{t \mid$ $\left.v_{\epsilon}(t)>0\right\}$, and $\mathcal{N}^{\varepsilon}=\left\{t \mid v^{\epsilon}(t)>0\right\}$. Then the following estimate holds

$$
\operatorname{meas} \mathcal{N}^{\varepsilon} \leq \frac{1}{\delta \varepsilon}\left(\int_{0}^{T} r v \mathrm{~d} t+\delta \int_{\mathcal{M}_{\delta}} v \mathrm{~d} t\right) .
$$

Proof. Since $\mathcal{N}^{\varepsilon} \subset \mathcal{N} \subset \mathcal{M}=\mathcal{M}_{\delta} \cup \mathcal{M}^{\delta}$, we have $\mathcal{N}^{\varepsilon}=\left(\mathcal{N}^{\varepsilon} \cap \mathcal{M}^{\delta}\right) \cup\left(\mathcal{N}^{\varepsilon} \cap \mathcal{M}_{\delta}\right)$. Consequently,

$$
\delta \varepsilon \text { meas } \mathcal{N}^{\varepsilon}=\int_{\mathcal{N}^{\varepsilon} \cap \mathcal{M}^{\delta}} \delta \varepsilon \mathrm{d} t+\int_{\mathcal{N}^{\varepsilon} \cap \mathcal{M}_{\delta}} \delta \varepsilon \mathrm{d} t \leq \int_{0}^{T} r v \mathrm{~d} t+\delta \int_{\mathcal{M}_{\delta}} v \mathrm{~d} t .
$$

The required estimate follows.

Proof of Lemma 8.1. Without loss of generality, assume that $\{t \mid v(t)>0\} \subset \mathcal{M}$ for all members of the sequence $\{v\}$. According to the conditions of the lemma, we have $\|v\|_{2} \leq \alpha$ and $\int_{0}^{T} r v \mathrm{~d} t \leq \rho \alpha$, where $\rho \rightarrow+0$. Set $\delta=\sqrt{\rho}$ and $\varepsilon=\alpha\left(\sqrt{\rho}+\sqrt{\text { meas } \mathcal{M}_{\delta}}\right)^{\frac{1}{2}}$, where $\mathcal{M}_{\delta}$ was defined in Proposition 10.7. Obviously, meas $\mathcal{M}_{\delta} \rightarrow 0$ and hence $\left\|v_{\varepsilon}\right\|_{\infty} \leq \varepsilon=o(\alpha)$. Since $\|v\|_{2} \leq \alpha$, according to Proposition 10.7 we have

$$
\begin{aligned}
\operatorname{meas}\left\{t \mid v^{\varepsilon}(t)>0\right\} & \leq \frac{1}{\delta \varepsilon}\left(\int_{0}^{T} r v \mathrm{~d} t+\delta \int_{\mathcal{M}_{\delta}} v \mathrm{~d} t\right) \\
& \leq \frac{1}{\delta \varepsilon}\left(\rho \alpha+\delta \sqrt{\text { meas } \mathcal{M}_{\delta}}\|v\|_{2}\right) \leq \frac{\alpha}{\delta \varepsilon}\left(\rho+\delta \sqrt{\text { meas } \mathcal{M}_{\delta}}\right) \\
& \leq\left(\sqrt{\rho}+\sqrt{\text { meas } \mathcal{M}_{\delta}}\right)^{\frac{1}{2}} \rightarrow 0 .
\end{aligned}
$$

Thus, it suffices to set $B=\left\{t \mid v^{\varepsilon}(t)>0\right\}$. The lemma is proved. 


\subsection{Proof of Lemma 8.2}

Assume that the assertion of the lemma is not true, i.e., there exist a sequence $\left\{u^{\prime}\right\} \in S^{\prime}$, a number $C^{\prime}<C$, and an element $\lambda \in M(C \Gamma)$ such that

$$
\int_{0}^{T}\left(\left\langle\bar{H}_{u u}(w, p, a) u^{\prime}, u^{\prime}\right\rangle+a\left(g^{\prime}(u) u^{\prime}\right)_{-}\right) \mathrm{d} t \leq C^{\prime} \int_{0}^{T}\left|u^{\prime}\right|^{2} \mathrm{~d} t
$$

for all members of the sequence. Since $\left\{u^{\prime}\right\}$ satisfies (8.1), the following estimates hold

$$
g\left(u+u^{\prime}\right) \leq k_{1}\left|u^{\prime}\right|^{2}, \quad\left|g_{j}\left(u+u^{\prime}\right)\right| \chi_{\left\{\hat{a}_{j} \geq \varepsilon\right\}} \leq k_{1}\left|u^{\prime}\right|^{2}, \quad j=1, \ldots, q,
$$

where $\varepsilon \rightarrow+0$ and $k_{1}>0$ does not depend on the member of the sequence. Due to the hypothesis of linear independence of gradients $g_{j}^{\prime}(u)$, there exists a sequence of corrections $\{v\}$ such that

$$
g\left(u+u^{\prime}+v\right) \leq 0, \quad g_{j}\left(u+u^{\prime}+v\right) \chi_{\left\{\hat{a}_{j} \geq \varepsilon\right\}}=0, \quad|v| \leq k_{2}\left|u^{\prime}\right|^{2},
$$

where $k_{2}>0$ does not depend on the member of the sequence. Set $\{\delta u\}=\left\{u^{\prime}+v\right\}$. Then we obviously have

$$
|\delta u|^{2}=\left|u^{\prime}\right|^{2}+r_{1},\left\langle\bar{H}_{u u}(w, p, a) \delta u, \delta u\right\rangle=\left\langle\bar{H}_{u u}(w, p, a) u^{\prime}, u^{\prime}\right\rangle+r_{2},
$$

where $\left|r_{i}\right| \leq k_{3}\left|u^{\prime}\right|^{3}, i=1,2$, and $k_{3}>0$ does not depend on the member of the sequence. Moreover, using the second and the third relations in (10.35), as well as inequalities (5.16), we get for any $j=1, \ldots, q$

$$
a_{j} g_{j}(u+\delta u)_{-}=a_{j} g_{j}(u+\delta u)_{-} \chi_{\left\{\hat{a}_{j}<\varepsilon\right\}} \leq a_{j}\left(g_{j}^{\prime}(u) u^{\prime}\right)_{-}+r_{4 j},
$$

where $\left|r_{4 j}\right| \leq \varepsilon k_{4 j}\left(|\delta u|^{2}+|v|\right) \leq \varepsilon k_{5 j}\left|u^{\prime}\right|^{2}$, and $k_{4 j}>0$ and $k_{5 j}>0$ does not depend on the member of the sequence. Consequently,

$$
\begin{aligned}
\int_{0}^{T}|\delta u|^{2} \mathrm{~d} t & =\int_{0}^{T}\left|u^{\prime}\right|^{2} \mathrm{~d} t+o\left(\gamma^{\prime}\right), \\
\int_{0}^{T}\left\langle\bar{H}_{u u}(w, p, a) \delta u, \delta u\right\rangle \mathrm{d} t & =\int_{0}^{T}\left\langle\bar{H}_{u u}(w, p, a) u^{\prime}, u^{\prime}\right\rangle \mathrm{d} t+o\left(\gamma^{\prime}\right), \\
\int_{0}^{T} a_{j} g_{j}(u+\delta u)_{-} \mathrm{d} t & \leq \int_{0}^{T} a_{j}\left(g_{j}^{\prime}(u) u^{\prime}\right)_{-} \mathrm{d} t+o\left(\gamma^{\prime}\right),
\end{aligned}
$$

where $\gamma^{\prime}=\int_{0}^{T}\left|u^{\prime}\right|^{2} \mathrm{~d} t$. These relations imply the inequality

$$
\int_{0}^{T}\left(\left\langle\bar{H}_{u u}(w, p, a) \delta u, \delta u\right\rangle+a \delta g_{-}\right) \mathrm{d} t \leq \int_{0}^{T}\left(\left\langle\bar{H}_{u u}(w, p, a) u^{\prime}, u^{\prime}\right\rangle+a\left(g^{\prime}(u) u^{\prime}\right)_{-}\right) \mathrm{d} t+o\left(\gamma^{\prime}\right),
$$

where $\delta g_{-}=g_{-}(u+\delta u)-g_{-}(u)$. Since $\bar{H}_{u}(w, p, a)=0, \delta_{u} \bar{H}=\delta_{u} H+a \delta g$, and $\delta g+\delta g_{-}=0$, where $\delta_{u} \bar{H}=\bar{H}(u+\delta u, y, p, a)-\bar{H}(u, y, p, a)$, etc., we obtain

$$
\begin{aligned}
\int_{0}^{T}\left(\left\langle\bar{H}_{u u}(w, p, a) \delta u, \delta u\right\rangle+a \delta g_{-}\right) \mathrm{d} t & =\int_{0}^{T}\left(\delta_{u} \bar{H}+a \delta g_{-}\right) \mathrm{d} t+o\left(\int_{0}^{T}|\delta u|^{2} \mathrm{~d} t\right) \\
& =\int_{0}^{T} \delta_{u} H \mathrm{~d} t+o\left(\int_{0}^{T}|\delta u|^{2} \mathrm{~d} t\right) .
\end{aligned}
$$


Combining relations (10.34), (10.37), (10.40), and (10.41), we get

$$
\int_{0}^{T} \delta_{u} H \mathrm{~d} t+o\left(\int_{0}^{T}|\delta u|^{2} \mathrm{~d} t\right) \leq C^{\prime} \int_{0}^{T}|\delta u|^{2} \mathrm{~d} t .
$$

But since the sequence $\{\delta u\}$ satisfies the condition $g(u+\delta u) \leq 0$ and $\lambda \in M(C \Gamma)$, we have $\delta_{u} H \geq C|\delta u|^{2}$, and hence $\int_{0}^{T} \delta_{u} H \mathrm{~d} t \geq C \int_{0}^{T}|\delta u|^{2} \mathrm{~d} t$. This together with estimate (10.42) contradicts the inequality $C>C^{\prime}$. The lemma is proved.

\subsection{Proof of Lemma 8.3}

Let $\{\delta w\} \in S_{1}$ and let $\{\varepsilon\}$ be the corresponding sequence of positive numbers converging to zero (see (6.15)). For any $j=1, \ldots, q$, define a sequence of sets $B_{j}^{0}=\left\{t \mid 0<\hat{a}_{j}(t)<\varepsilon\right\}$. Let $B^{0}=\bigcup_{j=1}^{q} B_{j}^{0}, \delta u_{B^{0}}=\delta u \chi_{B^{0}}$ and $\delta u^{0}=\delta u-\delta u_{B^{0}}=\delta u\left(1-\chi_{B^{0}}\right)$, where $\chi_{B^{0}}$ is the characteristic function of the set $B^{0}$. Note that $\left\|\delta u^{0}\right\|_{2} \leq$ $\|\delta u\|_{2} \leq \sqrt{\gamma(\delta w)}$. Since meas $B^{0} \rightarrow 0$, we have $\left\|\delta u_{B^{0}}\right\|_{1} \leq \sqrt{\text { meas } B^{0}}\|\delta u\|_{2}=o(\sqrt{\gamma(\delta w)})$. Moreover, we have $g_{j}^{\prime}(u) \delta u^{0} \chi_{\left\{g_{j}(u)=0\right\}} \leq 0, \hat{a}_{j} g_{j}^{\prime}(u) \delta u^{0}=0, j=1, \ldots, q$. Let us define a sequence $\left\{\delta y^{0}\right\}$ such that all members of the sequence $\left\{\delta w^{0}\right\}=\left\{\left(\delta u^{0}, \delta y^{0}\right)\right\}$ belong to the cone $\mathcal{R}_{0}$ (see (7.5)). From (6.5) we get $\delta \dot{y}=f_{y}(w) \delta y+f_{u}(w) \delta u+\zeta$, where $\|\zeta\|_{1}=o(\sqrt{\gamma(\delta w)})$. Define $\delta y^{0}$ as the solution to the equation $\delta \dot{y}^{0}=f_{y}(w) \delta y^{0}+f_{u}(w) \delta u^{0}, \delta y^{0}(0)=\delta y(0)$. Set $\bar{y}=\delta y-\delta y^{0}$. Then $\bar{y}$ satisfies the equation $\dot{\bar{y}}=f_{y}(w) \bar{y}+f_{u}(w) \delta u_{B^{0}}+\zeta, \bar{y}(0)=0$, from which, in view of estimates $\left\|\delta u_{B^{0}}\right\|_{1}=o(\sqrt{\gamma(\delta w)})$ and $\|\zeta\|_{1}=o(\sqrt{\gamma(\delta w)})$, it follows that $\|\bar{y}\|_{1,1}=o(\sqrt{\gamma(\delta w)})$. This and the estimate $\left\langle w_{1}^{*}, \delta w\right\rangle:=\varphi_{\eta}^{\mu^{1}}(\eta) \delta \eta \leq o(\sqrt{\gamma(\delta w)})$ imply $\left\langle w_{1}^{*}, \delta w^{0}\right\rangle \leq o(\sqrt{\gamma(\delta w)})$. But now $\delta w^{0} \in \mathcal{R}_{0}$, therefore we get $-\int_{0}^{T} a^{1} g^{\prime}(u) \delta u^{0} \mathrm{~d} t \leq o(\sqrt{\gamma(\delta w)})$. Since $\left\|\delta u_{B^{0}}\right\|_{1}=o(\sqrt{\gamma(\delta w)})$, we also get $-\int_{0}^{T} a^{1} g^{\prime}(u) \delta u \mathrm{~d} t \leq o(\sqrt{\gamma(\delta w)})$.

Now, using Lemma 8.1, let us change the members of the sequence $\left\{\delta u^{0}\right\}$ on the sets $\mathcal{M}_{j}^{1}:=\left\{t \mid a_{j}^{1}(t)>\right.$ $0\}, j=1, \ldots, q$, and define a new sequence $\left\{\delta w^{1}\right\}$ whose members belong to the cone $\mathcal{R}_{1}$. Set $v_{j}^{0}=$ $-g_{j}^{\prime}(u) \delta u^{0} \chi_{\left\{g_{j}(u)=0\right\}}, j=1, \ldots, q$. Then we have $v_{j}^{0} \geq 0,\left\|v_{j}^{0}\right\|_{2} \leq O(\sqrt{\gamma(\delta w)})$ and $\int_{0}^{T} a^{1} v_{j}^{0} \mathrm{~d} t=o(\sqrt{\gamma(\delta w)})$, $j=1, \ldots, q$. According to Lemma 8.1, for any $j$ there exist two sequences of sets $\left\{A_{j}^{1}\right\}$ and $\left\{B_{j}^{1}\right\}$ such that $A_{j}^{1} \cup B_{j}^{1}=\mathcal{M}_{j}^{1}, A_{j}^{1} \cap B_{j}^{1}=\emptyset$, meas $B_{j}^{1} \rightarrow 0$, and $\left\|v_{j}^{0} \chi_{A_{j}^{1}}\right\|_{\infty}=o(\sqrt{\gamma(\delta w)})$. Let $B^{1}=\bigcup_{j=1}^{q} B_{j}^{1}$ and $\delta u_{B^{1}}^{0}=\delta u^{0} \chi_{B^{1}}$. Since meas $B^{1} \rightarrow 0$ and $\left\|\delta u^{0}\right\|_{2} \leq \sqrt{\gamma(\delta w)}$ we have $\left\|\delta u_{B^{1}}^{0}\right\|_{1}=o(\sqrt{\gamma(\delta w)})$. In view of the hypothesis of linear independence of the gradients $g_{j}^{\prime}(u)$, from the estimate $\left\|v_{j}^{0} \chi_{A_{j}^{1}}\right\|_{\infty}=o(\sqrt{\gamma(\delta w)})$ it follows that there exists a sequence $\left\{\bar{u}^{0}\right\}$ such that $\left\|\bar{u}^{0}\right\|_{\infty}=o(\sqrt{\gamma(\delta w)}), \bar{u}^{0} \chi_{B^{1}}=0, g_{j}^{\prime}(u) \bar{u}^{0}=v_{j}^{0}$ a.e. on the set $A_{j}^{1} \backslash B^{1}$, and $g_{j}^{\prime}(u) \bar{u}^{0}=0$ a.e. on the set $\left\{t \mid g_{j}(u(t))=0\right\} \backslash \mathcal{M}_{j}^{1}, j=1, \ldots, q$. Set $\delta u^{1}=\delta u^{0}-\delta u_{B^{1}}^{0}-\bar{u}^{0}$ and define $\delta y^{1}$ as the solution to the equation $\delta \dot{y}^{1}=f_{y}(w) \delta y^{1}+f_{u}(w) \delta u^{1}, \delta y^{1}(0)=\delta y(0)$. Then, as it is easily seen, all members of the sequence $\left\{\delta w^{1}\right\}=\left\{\left(\delta u^{1}, \delta y^{1}\right)\right\}$ belong to the cone $\mathcal{R}_{1}$, and, for this sequence, we have $\left\langle w_{2}^{*}, \delta w^{1}\right\rangle \leq o(\sqrt{\gamma(\delta w)})$. This implies that $-\int_{0}^{T} a^{2} g^{\prime}(u) \delta u^{1} \mathrm{~d} t \leq o(\sqrt{\gamma(\delta w)})$, and then $-\int_{0}^{T} a^{2} g^{\prime}(u) \delta u \mathrm{~d} t \leq o(\sqrt{\gamma(\delta w)})$. Similarly, we change the sequence $\left\{\delta u^{1}\right\}$ on the sets $\mathcal{M}_{j}^{2}=\left\{t \mid a_{j}^{2}(t)>0\right\}$, $j=1, \ldots, q$, and deduce that $-\int_{0}^{T} a^{2} g^{\prime}(u) \delta u \mathrm{~d} t \leq o(\sqrt{\gamma(\delta w)})$, etc. The lemma is proved.

\section{HOFFMAN'S ERROR BOUNDS FOR A SYSTEM OF LINEAR INEQUALITIES ON A CONVEX CONE}

Formally this section is independent from the others. Following [18,20], pp. 229-243, in Sections 11.1-11.3 we study two questions concerning a system of linear inequalities on a convex cone: (a) the existence of a solution to the system; (b) the existence of Hoffman's type [7] upper bounds for the distance from the origin to the set of solutions to the system. In Section 11.4 we consider the same questions for a system of linear inequalities and equalities on a cone. In Section 11.5, we formulate an abstract notion of support of the critical cone and prove that the system of inequalities defining this cone possesses a Hoffman's error bound on the support. The main 
result of Sections 11.1-11.3 (Lem. 11.6) was published in [18] with a short sketch of the proof. Section 11.5 was written in 2007.

\subsection{On the compatibility of a linear system on a cone}

Let $X$ be a linear space, $l_{i}: X \rightarrow \mathbb{R}, i=1, \ldots, k$ a set of linear functionals, $K$ a convex cone in $X$. Consider the following system:

We will write it briefly as

$$
\left\langle l_{i}, x\right\rangle+\xi_{i} \leq 0, \quad i=1, \ldots, k, \quad x \in K .
$$

$$
l(x)+\xi \leq 0, \quad x \in K
$$

where $l: X \rightarrow \mathbb{R}^{k}$ is a linear operator which corresponds to the set of linear functionals $l_{i}: X \rightarrow \mathbb{R}, i=1, \ldots, k$, and $\xi=\left(\xi_{1}, \ldots, \xi_{k}\right)^{*} \in \mathbb{R}^{k}$.

Set $\Omega=l(K)+\mathbb{R}_{+}^{k}$, where $l(K)$ is the image of the cone $K$ under the mapping $l: K \rightarrow \mathbb{R}^{k}$. It is clear that $\Omega$ is a convex cone in $\mathbb{R}^{k}$, and system (11.2) is compatible (i.e., has a solution) iff $(-\xi) \in \Omega$. In what follows, for any $A \subset \mathbb{R}$ the inequality $A \geq 0$ means that inf $A \geq 0$.

Lemma 11.1. Assume that the cone $\Omega$ is closed. Then system (11.2) is compatible iff the relations $\alpha \in \mathbb{R}_{+}^{k *}$, $\alpha l(K) \geq 0$ imply $\alpha \xi \leq 0$.

Proof. Let system (11.2) be incompatible, i.e., $-\xi \notin \Omega$. Since $\Omega$ is a convex and closed cone, there exits a vector $\alpha \in \mathbb{R}^{k *}$ such that $\alpha(-\xi)<0 \leq \alpha \Omega$, where $\alpha \Omega=\{\alpha \omega \mid \omega \in \Omega\}$. The inequality $\alpha \Omega \geq 0$ implies that $\alpha l(K) \geq 0$ and $\alpha \mathbb{R}_{+}^{k} \geq 0$. Consequently, $\alpha \geq 0$. Moreover, $\alpha \xi>0$. Thus, we obtain: if the relations $\alpha \in \mathbb{R}_{+}^{k *}, \alpha l(K) \geq 0$ imply that $\alpha \xi \leq 0$, then system (11.2) is compatible.

Vice versa, let system (11.2) be compatible, and let $x_{0}$ be a solution to this system. Let $\alpha \in \mathbb{R}^{k *}$ and $\alpha l(K) \geq 0$. Since $x_{0} \in K$, we have $\alpha l\left(x_{0}\right) \geq 0$. Consequently, $\alpha \xi \leq \alpha l\left(x_{0}\right)+\alpha \xi=\alpha\left(l\left(x_{0}\right)+\xi\right) \leq 0$. The lemma is proved.

If $K=L$, where $L$ is a subspace in $X$, then the cone $\Omega=l(L)+\mathbb{R}_{+}^{k}$ is closed. It follows from the fact that, in this case, $\Omega$ is a finite faced cone, since $\Omega$ is the sum of the subspace $l(L)$ and the finite faced cone $\mathbb{R}_{+}^{k}$. Moreover, the inequality $\alpha l(L) \geq 0$ is equivalent to the equality $\alpha l(L)=0$. Thus we obtain the following corollary of Lemma 11.1.

Corollary 11.2. Let $K=L$, where $L$ is a subspace in $X$. Then system (11.2) is compatible iff the relations $\alpha \in \mathbb{R}_{+}^{k *}, \alpha l(L)=0$ imply $\alpha \xi \leq 0$.

\subsection{On the existence of Hoffman's error bound on a cone}

Now let us study the second question: how one can estimate (from above) the distance from the origin to the set of solutions to system (11.1)? Although $X$ is assumed to be a linear space, any finite dimensional subspace $H \subset X$ can be endowed with a norm $\|\cdot\|$ and any two norms on $H$ are equivalent. The following definition pertains to this possibility.

Definition 11.3. We say that system (11.2) has Hoffman's error bound if there exists a finite dimensional subspace $H \subset X$ such that for any norm $\|\cdot\|$ in $H$ there exists a constant $N=N(l, K, H,\|\cdot\|)>0$ such that the following condition holds: if system (11.2) is compatible, then the system

$$
l(x)+\xi \leq 0, \quad x \in K \cap H
$$

is also compatible, and there exists a solution $x_{0}$ to system (11.3) such that

$$
\left\|x_{0}\right\| \leq N\left|\xi_{+}\right|
$$

where $\left|\xi_{+}\right|=\max _{1 \leq i \leq k} \xi_{i+}$, and $\xi_{i+}=\max \left\{\xi_{i}, 0\right\}$. 
The well-known Hoffman's lemma states that if $K=X$ and the space $X$ is finite dimensional, then system (11.2) has Hoffman's error bound.

So, we shall study the question: under what conditions system (11.2) has Hoffman's error bound? In the sequel, we do not assume that the space $X$ is finite dimensional.

Recall that a cone $C$ is called finite generated if there exists a finite set of its elements $a_{1}, \ldots, a_{s}$ (generators of the cone) such that each element $x \in C$ can be represented as $x=\lambda_{1} a_{1}+\ldots+\lambda_{s} a_{s}$ with $\lambda_{1} \geq 0, \ldots, \lambda_{s} \geq 0$. In a finite dimensional space, a cone is finite faced iff it is finite generated.

Hoffman's lemma has the following simple generalization.

Lemma 11.4. Assume that $\Omega=l(K)+\mathbb{R}_{+}^{k}$ is a finite faced cone. Then system (11.2) has Hoffman's error bound.

Proof. Since $\Omega$ is a finite faced cone in $\mathbb{R}^{k}$, then $\Omega$ is finite generated. Let $\xi^{i}=l\left(x^{i}\right)+\eta^{i}, i=1, \ldots, r$ be the generators of the cone $\Omega$, where $x^{i} \in K, \eta^{i} \in \mathbb{R}_{+}^{k}, i=1, \ldots, r$. Let $H$ be the linear span of the set $\left\{x^{1}, \ldots, x^{r}\right\}$, and let $K_{0}$ be the cone generated by $x^{1}, \ldots, x^{r}$. Then it is easy to see that $\Omega=l\left(K_{0}\right)+\mathbb{R}_{+}^{k}$. The cone $K_{0}$ is finite generated and hence finite faced in $H$. Consequently, there exist linear functionals $m_{j}: H \rightarrow \mathbb{R}, j=1, \ldots, s$ such that $K_{0}=\left\{x \in H \mid\left\langle m_{j}, x\right\rangle \leq 0, j=1, \ldots, s\right\}$. In the finite dimensional subspace $H$, let us consider the system

$$
\left\langle l_{i}, x\right\rangle+\xi_{i} \leq 0, \quad i=1, \ldots, k, \quad\left\langle m_{j}, x\right\rangle \leq 0, \quad j=1, \ldots, s, \quad x \in H .
$$

The set of solutions to system (11.5) contains in the set of solutions to system (11.1); moreover, both sets are empty or nonempty simultaneously. Now, using Hoffman's lemma for system (11.5), we obtain the required error bound. The lemma is proved.

Corollary 11.5. If $K=L$ is a subspace in $X$, then $\Omega=l(K)+\mathbb{R}_{+}^{k}$ is a finite faced cone, and hence system (11.2) has Hoffman's error bound.

\subsection{Special case, where Hoffman's error bound holds}

Lemma 11.6. Let $Y$ be a locally convex topological space, $X=Y^{*}$ the dual space, $K_{Y}$ a closed convex cone in $Y$, $K=K_{Y}^{*}$ the dual cone in $X, l_{i}, i=1, \ldots, k$ a set of elements of the space $Y$ considered as the linear functionals on $X$. Denote by $K_{l}$ the cone in $Y$ generated by the elements $l_{i}, i=1, \ldots, k$, i.e., $K_{l}=\left\{y=\alpha l \mid \alpha \in \mathbb{R}_{+}^{k *}\right\}$. Assume that there exists a subspace $S \subset Y$ such that $K_{l} \cap K_{Y} \subset S \subset K_{Y}$. Then system (11.2) has Hoffman's error bound. Moreover, system (11.2) is compatible iff the conditions $\alpha \in \mathbb{R}_{+}^{k *}, \alpha l \in K_{Y}$ imply that $\alpha \xi \leq 0$. In particular, for any $x_{0} \in K$ the system $l\left(x+x_{0}\right) \leq 0, x \in K$ is always compatible.

The proof of this lemma will be based on the following theorem.

Theorem 11.7. Let $Y$ be a locally convex topological space, $K_{1} \subset Y$ a finite generated cone, $K_{2} \subset Y$ a convex closed cone. Assume that there exists a subspace $S \subset Y$ such that $K_{1} \cap K_{2} \subset S \subset K_{2}$. Then $\left(K_{1} \cap K_{2}\right)^{*}=$ $K_{1}^{*}+K_{2}^{*}$.

Proof. (a) First, consider the case $K_{1} \cap K_{2}=S$, where $S$ is a subspace in $Y$. Set $Q=K_{1}^{*}+K_{2}^{*}$. We must show that $Q=S^{*}$.

It is obvious, that $S^{*}=\left(K_{1} \cap K_{2}\right)^{*} \supset K_{1}^{*}+K_{2}^{*}=Q$. Next, it is easy to see that, for two nonempty cones, the dual cone to their sum is equal to the intersection of their dual cones. Therefore, $Q^{*}=\left(K_{1}^{*}+K_{2}^{*}\right)^{*}=$ $K_{1}^{* *} \cap K_{2}^{* *}$. Since $K_{1}$ is a finite generated cone, we have $K_{1}^{* *}=K_{1}$. Moreover, $K_{1} \subset Y$. Consequently, $Q^{*}=K_{1} \cap K_{2}^{* *}=K_{1} \cap\left(K_{2}^{* *} \cap Y\right)$. Since $K_{2}$ is a convex closed cone and $Y$ is a locally convex topological space, we have $K_{2}^{* *} \cap Y=K_{2}$ (here, local convexity of $Y$ was used). Thus we obtain $Q^{*}=K_{1} \cap K_{2}=S$. Moreover, $Q \subset S^{*}$. Let us show that this implies the equality $Q=S^{*}$.

Assume that $Q \subset S^{*}$ and $Q \neq S^{*}$. Then there exists $x \in S^{*}$ such that $x \notin Q$. Consequently, there exists $x^{*} \in Q^{*}$ such that $\left\langle x^{*}, x\right\rangle<0$. But since $x^{*} \in Q^{*}, Q^{*}=S$, and $x \in S^{*}$, we have $\left\langle x^{*}, x\right\rangle \geq 0$. This contradiction proves that the condition $Q \neq S^{*}$ does not hold, i.e., $Q=S^{*}$. 
(b) Now, consider the general case: $K_{1} \cap K_{2} \subset S \subset K_{2}$, where $S$ is a subspace in $Y$. Since the cone $K_{1}$ is finite dimensional, without loss of generality, we can assume that the subspace $S$ is finite dimensional.

Set $\hat{K}_{1}=K_{1}+S$. Let us show that $\hat{K}_{1} \cap K_{2}=S$. Since $0 \in K_{1}$, we have $S \subset \hat{K}_{1}$. Moreover, $S \subset K_{2}$. Therefore, $S \subset \hat{K}_{1} \cap K_{2}$. Vice versa, let $y_{2} \in K_{2}$ and $y_{2} \in \hat{K}_{1}=K_{1}+S$, i.e. $y_{2}=y_{1}+s$, where $y_{1} \in K_{1}$ and $s \in S$. Since $S$ is a subspace, we have $-s \in S$. Therefore, $-s \in K_{2}$. Since $K_{2}$ is a convex cone, we have $y_{1}=y_{2}-s \in K_{2}$. Consequently, $y_{1} \in K_{1} \cap K_{2} \subset S$. Therefore, $y_{2}=y_{1}+s \in S$. This implies that $\hat{K}_{1} \cap K_{2} \subset S$. Consequently, $\hat{K}_{1} \cap K_{2}=S$.

Since $K_{1} \cap K_{2} \subset S \subset K_{2}$, we have $K_{1} \cap K_{2}=K_{1} \cap S$. The cone $K_{1}$ is finite generated, and $S$ is a finite dimensional subspace, hence $S$ can be also considered as a finite generated cone. As it is well-known for finite generated cones, the dual cone to their intersection is equal to the sum of their dual cones. Therefore, $\left(K_{1} \cap K_{2}\right)^{*}=\left(K_{1} \cap S\right)^{*}=K_{1}^{*}+S^{*}$. Further, since $\hat{K}_{1} \cap K_{2}=S \subset K_{2}$ and the cone $\hat{K}_{1}=K_{1}+S$ is finite generated, in virtue of (a) we have $S^{*}=\left(\hat{K}_{1} \cap K_{2}\right)^{*}=\hat{K}_{1}^{*}+K_{2}^{*}$. But $\hat{K}_{1}^{*}=\left(K_{1}+S\right)^{*}=K_{1}^{*} \cap S^{*}$. Consequently, $S^{*}=K_{1}^{*} \cap S^{*}+K_{2}^{*}$. Thus, we get $\left(K_{1} \cap K_{2}\right)^{*}=K_{1}^{*}+S^{*}=K_{1}^{*}+\left(K_{1}^{*} \cap S^{*}\right)+K_{2}^{*}=K_{1}^{*}+K_{2}^{*}$. The theorem is proved.

This theorem was discussed and proved in the seminar of Milyutin.

Proof of Lemma 11.6. Set $C=\left(K_{l} \cap K_{Y}\right)^{*}, \Omega=l(K)+\mathbb{R}_{+}^{k}$. Let us show that $\Omega=l(C)+\mathbb{R}_{+}^{k}$. Indeed, in virtue of Theorem 11.7, $C=K_{l}^{*}+K_{Y}^{*}$. Consequently, $l(C)=l\left(K_{l}^{*}\right)+l\left(K_{Y}^{*}\right)$. But, obviously, $l\left(K_{l}^{*}\right) \subset \mathbb{R}_{+}^{k}$, and hence $l\left(K_{l}^{*}\right)+\mathbb{R}_{+}^{k}=\mathbb{R}_{+}^{k}$. Consequently, $l(C)+\mathbb{R}_{+}^{k}=l\left(K_{Y}^{*}\right)+\mathbb{R}_{+}^{k}=l(K)+\mathbb{R}_{+}^{k}=\Omega$. Further, as it was mentioned in the proof of Theorem 11.7, $K_{l} \cap K_{Y}=K_{l} \cap S$. Since $K_{l}$ is a finite generated cone and $S$ is a subspace, the cone $K_{l} \cap S$ is finite generated too. Consequently, $C=\left(K_{l} \cap K_{Y}\right)^{*}$ is a finite faced cone in $X$. This implies that $\Omega=l(C)+\mathbb{R}_{+}^{k}$ is a finite faced cone too.

Since each finite faced cone is closed, by Lemma 11.1 the compatibility of the system $l(x)+\xi \leq 0, x \in K$ is equivalent to the condition: if $\alpha \in \mathbb{R}_{+}^{k}, \alpha l(K) \geq 0$, then $\alpha \xi \leq 0$. But since $l_{i} \in Y$ for all $i$, the condition $\alpha l(K) \geq 0$ is equivalent to the condition $\alpha l \in K^{*} \cap Y=K_{Y}$. This implies the assertion of the lemma concerning the compatibility of the system. In particular, if $x_{0} \in K$ and $\xi_{0}=l\left(x_{0}\right)$, then the conditions $\alpha l \in K_{Y}, \alpha \in \mathbb{R}_{+}^{k}$ imply $\alpha l \in S \subset K_{Y}$. Since $S$ is a subspace, $K=K_{Y}^{*}$, and $x_{0} \in K$, we have $\alpha l\left(x_{0}\right)=0$, i.e., $\alpha \xi_{0}=0$. Thus, in this case the system is compatible. The lemma is proved.

\subsection{System of linear inequalities and equalities on a cone}

Let $Y$ be a locally convex topological space, $X=Y^{*}$ the dual space, $K_{Y}$ a closed convex cone in $Y, K=K_{Y}^{*}$ the dual cone in $X, l_{i}, i=1, \ldots, k$ and $m_{j}, j=1, \ldots, s$ two sets of elements of the space $Y$ considered as the linear functionals on $X$. Let $l: X \rightarrow \mathbb{R}^{k}$ and $m: X \rightarrow \mathbb{R}^{s}$ be the linear operators corresponding to the sets of functionals $l_{i}, i=1, \ldots, k$ and $m_{j}, j=1, \ldots, s$, respectively. Consider the system of linear inequalities and equalities on the cone $K$ :

where $\xi \in \mathbb{R}^{k}$ and $\eta \in \mathbb{R}^{s}$.

$$
l(x)+\xi \leq 0, m(x)+\eta=0, x \in K,
$$

Definition 11.8. We say that system (11.6) has Hoffman's error bound if there exists a finite dimensional subspace $H \subset X$ such that for any norm $\|\cdot\|$ in $H$ there exists a constant $N>0$ such that the following condition holds: if system (11.6) is compatible, then the system

$$
l(x)+\xi \leq 0, m(x)+\eta=0, x \in K \cap H
$$

is compatible too, and there exists a solution $x$ to system (11.7) such that

$$
\|x\| \leq N\left(\left|\xi_{+}\right|+|\eta|\right) .
$$

Denote by $K_{l}$ the cone in $Y$ generated by the elements $l_{i}, i=1, \ldots, k$, and denote by $L_{m}$ the subspace generated by the elements $m_{j}, j=1, \ldots, k$. Set $K_{l m}=K_{l}+L_{m}$, i.e., $K_{l m}=\left\{y=\alpha l+\beta m \mid \alpha \in \mathbb{R}_{+}^{k *}, \beta \in \mathbb{R}^{s *}\right\}$. 
Lemma 11.9. Assume that there exists a subspace $S \subset Y$ such that $K_{l m} \cap K_{Y} \subset S \subset K_{Y}$. Then system (11.6) has Hoffman's error bound. Moreover, system (11.6) is compatible iff the conditions $\alpha \in \mathbb{R}_{+}^{k *}, \beta \in \mathbb{R}^{s *}, \alpha l+$ $\beta m \in K_{Y}$ imply $\alpha \xi+\beta \eta \leq 0$. In particular, for any $x_{0} \in K$, the system $l\left(x+x_{0}\right) \leq 0, m\left(x+x_{0}\right)=0, x \in K$ is always compatible.

Proof. System (11.6) can be represented as a system of inequalities

$$
l(x)+\xi \leq 0, m(x)+\eta \leq 0,-m(x)-\eta \leq 0, x \in K,
$$

and hence one can apply Lemma 11.6 to the latter system. Obviously, system (11.6) is compatible iff system (11.9) is compatible; moreover system (11.6) has Hoffman's error bound iff system (11.9) has Hoffman's error bound. Thus we get an analog of Lemma 11.6 for system (11.6).

\subsection{Abstract notion of support of critical cone}

Let $Y$ be a Banach space, $X=Y^{*}$ a dual space, $l_{i}, i=1, \ldots, k$ a set of elements in the space $Y$ considered as linear functionals on $X, C$ a closed convex cone in $Y, \Omega=C^{*}$ a dual cone in $X$. Set $K=\{x \in \Omega \mid$ $\left.l_{i}(x) \leq 0, i=1, \ldots, k\right\}$. The cone $K$ will serve us as an abstract model of the critical cone (see, e.g., [6]). We shall define the notion of the support of the cone $K$. Set $Q=\left\{l=\sum_{i=1}^{k} \alpha_{i} l_{i} \mid \alpha_{i} \geq 0, i=1, \ldots, k\right\}$. For any $y \in Q$ and for any $x \in K$ we obviously have: $\langle y, x\rangle \leq 0$.

Let $y_{1} \in Q$ be such that $y_{1}(\Omega) \geq 0$ (i.e. $y_{1} \in C$ ) and let $x_{1} \in \Omega$ be such that $\left\langle y_{1}, x_{1}\right\rangle>0$. Set $\Omega_{1}=\{x \in \Omega \mid$ $\left.\left\langle y_{1}, x\right\rangle=0\right\}$. Then $K \subset \Omega_{1}$. Moreover, the cone $\Omega_{1}$ is dual to the cone $C_{1}:=C+\operatorname{Span}\left\{y_{1}\right\}$, where $\operatorname{Span}\left\{y_{1}\right\}$ is a one dimensional subspace generated by vector $y_{1}$. Indeed, $\left(C+\operatorname{Span}\left\{y_{1}\right\}\right)^{*}=C^{*} \cap \operatorname{Span}\left\{y_{1}\right\}^{*}=\Omega \cap\{x \in$ $\left.X \mid\left\langle y_{1}, x\right\rangle=0\right\}=\Omega_{1}$.

Let $y_{2} \in Q$ be such that $y_{2}\left(\Omega_{1}\right) \geq 0$ (i.e. $y_{2} \in C_{1}$ ) and let $x_{2} \in \Omega_{1}$ be such that $\left\langle y_{2}, x_{2}\right\rangle>0$. Set $\Omega_{2}=\left\{x \in \Omega_{1} \mid\left\langle y_{2}, x\right\rangle=0\right\}$. Then the cone $\Omega_{2}$ is dual to the cone $C_{2}:=C_{1}+\operatorname{Span}\left\{y_{2}\right\}$.

Assume that we have already cones $\Omega_{1}, \Omega_{2}, \ldots, \Omega_{s-1}$, dual to the cones $C_{1}, C_{2}, \ldots, C_{s-1}$, respectively, and functionals $y_{1} \in Q \cap C, y_{2} \in Q \cap C_{1}, \ldots, y_{s-1} \in Q \cap C_{s-2}$. Let $y_{s} \in Q$ be such that $y_{s}\left(\Omega_{s-1}\right) \geq 0$ (i.e. $\left.y_{s} \in C_{s-1}\right)$ and let $x_{s} \in \Omega_{s-1}$ be such that $\left\langle y_{s}, x_{s}\right\rangle>0$. Set $\Omega_{s}=\left\{x \in \Omega_{s-1} \mid\left\langle y_{s}, x\right\rangle=0\right\}$. Then the cone $\Omega_{s}$ is dual to the cone $C_{s}:=C_{s-1}+\operatorname{Span}\left\{y_{s}\right\}$. Let us show that the vectors $y_{1}, \ldots, y_{s}$ are linearly independent. Let the numbers $\lambda_{1}, \ldots, \lambda_{s-1}$ be such that $y_{s}=\lambda_{1} y_{1}+\ldots+\lambda_{s-1} y_{s-1}$. Since $y_{k}\left(\Omega_{k}\right) \equiv 0, k=1, \ldots, s-1$ and $\Omega_{s} \subset \Omega_{s-1} \subset \ldots \subset \Omega_{1} \subset \Omega$, we have $y_{k}\left(\Omega_{s-1}\right) \equiv 0, k=1, \ldots, s-1$, and hence $y_{s}\left(\Omega_{s-1}\right) \equiv 0$. This is a contradiction with condition $\left\langle y_{s}, x_{s}\right\rangle>0$. Consequently, $y_{s} \notin \operatorname{Span}\left\{y_{1}, \ldots, y_{s-1}\right\}$. Since the vectors $y_{1}, \ldots, y_{s} \in Q$ are linearly independent and the set $Q$ is finite dimensional, this process will be finished on a certain step $s$.

Let the system $y_{1}, \ldots, y_{s}$ be maximal. Then for the cone $\Omega_{s}$ we have: if $y \in Q$ is such that $y\left(\Omega_{s}\right) \geq 0$ (i.e. $\left.y \in C_{s}\right)$, then $y\left(\Omega_{s}\right) \equiv 0$. In this case we have $(-y) \in C_{s}$. It means that the linear span of the intersection $Q \cap C_{s}$ is a subspace in $C_{s}$. Denote this subspace by $H$. Set $\mathcal{S}=\Omega_{s}, \mathcal{S}_{Y}=C_{s}$. Then $\mathcal{S}_{Y}^{*}=\mathcal{S}, \mathcal{S}_{Y} \cap Q \subset H \subset \mathcal{S}_{Y}$, where $H$ is a subspace in the cone $\mathcal{S}_{Y}$. Thus, for the system of functionals $l_{1}, \ldots, l_{k}$ considered on the cone $\mathcal{S}$, the conditions of Lemma 11.6 are satisfied. By this lemma, for any $x_{0} \in \mathcal{S}$, the system $l\left(x+x_{0}\right) \leq 0, x \in \mathcal{S}$ is compatible and has Hoffman's error bound. We call $\mathcal{S}$ the support of the cone $K$.

Remark 11.10. As before, let $K=\left\{x \in \Omega \mid l_{i}(x) \leq 0, i=1, \ldots, k\right\}$. Denote by $\hat{l}_{i}, i=1, \ldots, \hat{k}$ all functionals of the set $l_{1}, \ldots, l_{k}$ such that $\hat{l}_{i}(K) \equiv 0$. All functionals $l_{i}$ which do not posses this property we denote by $\tilde{l}_{i}$, $i=1, \ldots, \tilde{k}$, where $\tilde{k}:=k-\hat{k}$. By definition, for each $\tilde{l}_{i}$ there exits an element $\tilde{x}_{i} \in K$ such that $\tilde{l}_{i}\left(\tilde{x}_{i}\right)<0$. Set $\tilde{x}=\sum \tilde{x}_{i}$. Then $\tilde{x} \in K$, and $\tilde{l}_{i}(\tilde{x})<0$ for all $i=1, \ldots, \tilde{k}$. Set $\hat{\Omega}=\left\{x \in \Omega \mid \hat{l}_{i}(x)=0 \forall i\right\}$. One can show that $K \subset \hat{\Omega} \subset \mathcal{S}$, and, for any $x_{0} \in \hat{\Omega}$, the system $l_{i}\left(x_{0}+x\right) \leq 0, i=1, \ldots, k, x \in \hat{\Omega}$ is compatible and has Hoffman's error bound. Question: is it true that $\hat{\Omega}=\mathcal{S}$ ? A simple example shows that this is not true. Let $k=2, l_{1}$ be such that $l_{1}(\Omega)=\mathbb{R}$, and $l_{2}=-l_{1}$. Then $\hat{l}_{1}=l_{1}, \hat{l}_{2}=l_{2}$ and $K=\hat{\Omega}=\left\{x \in \Omega \mid l_{1}(x)=0\right\} \neq \Omega=\mathcal{S}$. 
Acknowledgements. I obtained Theorem 4.1 about 30 years ago. At that time I had permanent contacts with my teacher A.A. Milyutin, and some of his ideas were used in the proof of this theorem. But (as it was mentioned in Introduction) this proof has never been published. The revision of the proof with its partial modification was mainly performed during my stay at École polytechnique, France, in 2007. I would like to express my gratitude to F. Bonnans for our numerous fruitful discussions which initiated this publication. Also I am thankful to anonymous referees for their valuable remarks.

\section{REFERENCES}

[1] J.F. Bonnans and A. Hermant, Second-order analysis for optimal control problems with pure state constraints and mixed control-state constraints. Ann. Inst. Henri Poincaré, Anal. Non Linéaire 26 (2009) 561-598.

[2] J.F. Bonnans and N.P. Osmolovskii, Second-order analysis of optimal control problems with control and initial-final state constraints. J. Convex Anal. 17 (2010) 885-913.

[3] J.F. Bonnans and A. Shapiro, Perturbation Analysis of Optimal Control Problems. Springer, New York (2000).

[4] A.V. Dmitruk, Quadratic conditions for a Pontryagin minimum in an optimal control problem linear in control. I. Deciphering theorem. Izv. Akad. Nauk SSSR 50 (1986) 284-312.

[5] A.V. Dmitruk, Quadratic conditions for a Pontryagin minimum in an optimal control problem linear in control. II. Theorem on weakening inequality constraints. Izv. Akad. Nauk SSSR 51 (1987) 812-832.

[6] A.Ya. Dubovitski and A.A. Milyutin, Extremum problems in the presence of restrictions. Zh. Vychislit. Mat. i Mat. Fiz. 5 (1965) 395-453; English translation in U.S.S.R. Comput. Math. Math. Phys. 5 (1965) 1-80.

[7] A.J. Hoffman, On approximate solutions of systems of linear inequalities. J. Res. Nat'l Bur. Standarts 49 (1952) 263-265.

[8] E.S. Levitin, A.A. Milyutin and N.P. Osmolovskii, Higher-order local minimum conditions in problems with constraints. Uspekhi Mat. Nauk 33 (1978) 85-148; English translation in Russian Math. Surveys 33 (1978) 97-168.

[9] K. Malanowski, Stability and sensitivity of solutions to nonlinear optimal control problems. Appl. Math. Optim. 32 (1994) $111-141$.

[10] K. Malanowski, Sensitivity analysis for parametric control problems with control-state constraints. Dissertationes Mathematicae CCCXCIV. Polska Akademia Nauk, Instytut Matematyczny, Warszawa (2001) 1-51.

[11] H. Maurer, First and second order sufficient optimality conditions in mathematical programming and optimal control. Mathematical Programming Study 14 (1981) 163-177.

[12] H. Maurer and S. Pickenhain, Second order sufficient conditions for optimal control problems with mixed control-state constraints. J. Optim. Theory Appl. 86 (1995) 649-667.

[13] A.A. Milyutin, Maximum Principle in the General Optimal Control Problem. Fizmatlit, Moscow (2001) [in Russian].

[14] A.A. Milyutin and N.P. Osmolovskii, High-order conditions for a minimum on a set of sequences in the abstract problem with inequality constraints. Comput. Math. Model. 4 (1993) 393-400.

[15] A.A. Milyutin and N.P. Osmolovskii, High-order conditions for a minimum on a set of sequences in the abstract problem with inequality and equality constraints. Comput. Math. Model. 4 (1993) 401-409.

[16] A.A. Milyutin and N.P. Osmolovskii, High-order conditions with respect to a subsystem of constraints in the abstract minimization problem on a set of sequences. Comput. Math. Model. 4 (1993) 410-418.

[17] A.A. Milyutin and N.P. Osmolovskii, Calculus of Variations and Optimal Control, Translations of Mathematical Monographs 180. American Mathematical Society, Providence (1998).

[18] N.P. Osmolovskii, On a system of linear inequalities on a convex set. Usp. Mat. Nauk. 32 (1977) $223-224$ [in Russian].

[19] N.P. Osmolovskii, Higher-Order Necessary and Sufficient Conditions in Optimal Control. Parts 1 and 2, Manuscript deposited in VINITI April 1, No. 2190-B and No. 2191-B (1986) [in Russian].

[20] N.P. Osmolovskii, Theory of higher order conditions in optimal control. Ph.D. thesis, Moscow (1988) [in Russian].

[21] N.P. Osmolovskii, Quadratic optimality conditions for broken extremals in the general problem of calculus of variations. $J$. Math. Sci. 123 (2004) 3987-4122.

[22] N.P. Osmolovskii, Sufficient quadratic conditions of extremum for discontinuous controls in optimal control problems with mixed constraints. J. Math. Science 173 (2011) 1-106.

[23] V. Zeidan, Extended Jacobi sufficiency criterion for optimal control. SIAM J. Control. Optim. 22 (1984) 294-301.

[24] V. Zeidan, The Riccati equation for optimal control problems with mixed state-control constraints: necessity and sufficiency. SIAM J. Control Optim. 32 (1994) 1297-1321. 